\title{
Nanomechanical architecture of semiconductor nanomembranes
}

\author{
Minghuang Huang, ${ }^{* a}$ Francesca Cavallo, ${ }^{a}$ Feng Liu ${ }^{b}$ and Max G. Lagally ${ }^{a}$ \\ Received 2nd September 2010, Accepted 13th September 2010 \\ DOI: $10.1039 / \mathrm{c0nr00648c}$
}

\begin{abstract}
Semiconductor nanomembranes are single-crystal sheets with thickness ranging from 5 to $500 \mathrm{~nm}$. They are flexible, bondable, and mechanically ultra-compliant. They present a new platform to combine bottom-up and top-down semiconductor processing to fabricate various three-dimensional (3D) nanomechanical architectures, with an unprecedented level of control. The bottom-up part is the self-assembly, via folding, rolling, bending, curling, or other forms of shape change of the nanomembranes, with top-down patterning providing the starting point for these processes. The self-assembly to form 3D structures is driven by elastic strain relaxation. A variety of structures, including tubes, rings, coils, rolled-up "rugs", and periodic wrinkles, has been made by such selfassembly. Their geometry and unique properties suggest many potential applications. In this review, we describe the design of desired nanostructures based on continuum mechanics modelling, definition and fabrication of 2D strained nanomembranes according to the established design, and release of the 2D strained sheet into a 3D or quasi-3D object. We also describe several materials properties of nanomechanical architectures. We discuss potential applications of nanomembrane technology to implement simple and hybrid functionalities.
\end{abstract}

${ }^{a}$ University of Wisconsin-Madison, Madison, WI, 53706, USA. E-mail: mhhuang@cae.wisc.edu

${ }^{b}$ University of Utah, Salt Lake City, UT, 84112, USA

\section{Introduction}

The nanotechnology era, born in the 1980s with the development of scanned-probe techniques to visualize structures at the subnanometer scale, has brought many innovations in materials and

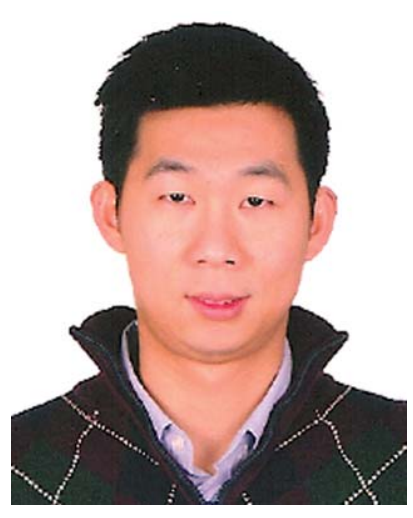

Minghuang Huang
Minghuang Huang is a scientist in the Materials Science and Engineering Department at the University of Wisconsin-Madison. His current research interests include nanomechanical architectures of strained Si/Ge nanomembranes and novel nanostructured materials for thermoelectric devices. He received his B.S. degree in Chemistry from the National Tsing-Hua University, Taiwan, R.O.C., and his M.S. and Ph.D. degrees in Materials Science and Engineering from the

University of Utah. He has been a process engineer at United Microelectronic Corporation (UMC), Hsinchu, Taiwan, R. O. C. and a senior engineer at Fairchild Semiconductor, Salt Lake City, Utah.

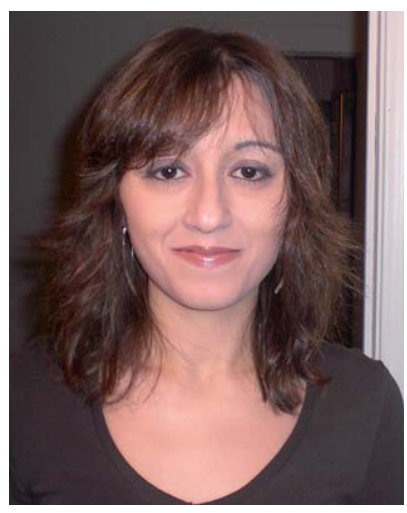

Francesca Cavallo
Francesca Cavallo is a postdoctoral fellow in the Materials Science and Engineering Department at the University of Wisconsin-Madison. Her research focuses on fabrication, modeling and characterization of semiconductor based nanostructures for highly integrative applications. She received her Ph.D. degree in Electrical Engineering and Information Technology with magna cum laude from the Technical University of Chemnitz (Germany). She conducted her research studies for her Ph.D. degree at the Max Planck Institute for Solid State Research (Stuttgart, Germany) and at Institute for Integrative Nanosciences (Dresden, Germany). She received her M.S. in Electrical Engineering with highest honors from the University of Salerno (Italy). 
processes. Those having the highest scientific impact were frequently based on self-assembly or "bottom-up" methods: quantum dot growth, ${ }^{1,2}$ nanowire, ${ }^{3,4}$ or nanotube growth, ${ }^{5}$ and DNA or other organic-molecule-based architectures. ${ }^{6}$ The difficulty with all these methods is, of course, that they are stochastic, and thus have a variation in nanostructural parameters like size, shape, position, and composition. Concurrently, "top-down", lithography-based processes, which can produce thousands of the same structures and do not suffer from stochasticity, have continued to make significant progress, but it has been obvious that a combination of these methods can provide powerful advances in nanotechnology and in understanding behaviors at the nanoscale. A new platform developed in the last decade, semiconductor nanomembranes, has significantly advanced the ability to combine bottom-up and top-down semiconductor processing to fabricate various three-dimensional (3D) nanoand micro-objects with an unprecedented level of control. ${ }^{7-20}$ The bottom-up part is the self-assembly, via folding, rolling, bending, curling, or other forms of shape change of the nanomembranes, with top-down processing providing the starting point for the self-assembly. The geometry, mechanical and thermal stability, and unique properties of these self-assembled nanomembranes have suggested new mechanical, ${ }^{12,13}$ optical, ${ }^{14,15}$ and electronic ${ }^{17}$ devices, as well as more complex hybrid functions such as drug delivery, ${ }^{18}$ micro-reactors, ${ }^{18}$ scaffolds for cell culture, ${ }^{19}$ and optofluidic components. ${ }^{20}$

Bending, rolling, or folding of nanometer-thick membranes is a response to the release of a large amount of internal potential energy in a constrained film upon the removal of the constraint. This potential energy can be stored in various ways, e.g., by mechanical stress, by surface tension, by a magnetic or electric field, or by chemical modification of the surfaces of the membrane.

We review here stress-driven formation of $3 \mathrm{D}$ and quasi-3D micro- and nanomechanical architectures. Nature provides many examples of stress-driven actuation. The Venus flytrap, for example, has a simple system based on hydrostatic pressure and release of stored elastic energy. ${ }^{21}$ When resting, the two leaves of the hinged trap are open in a concave shape to admit unsuspecting insects. When an intruding fly touches trigger hairs inside the trap, movement of fluid within leaf cells builds up elastic strain in the leaves. When the leaves can no longer sustain the strain, they snap into their other stable shape, curving inward to form a concave enclosure. When fruits or vegetables dry out the turgor pressure is relieved and the tissue starts to wrinkle; on the other hand, when they are preserved in a humid environment cracks form as a result of the increase of the turgor by transpiration. Corrugation and cracking of thin films are other examples of stress-driven phenomena. The delamination of vacuumdeposited metal thin films is caused by large internal stresses arising during the deposition. ${ }^{22,23} \mathrm{~A}$ significant effort has been directed to minimizing the internal stress of deposited thin films, ${ }^{24}$ because typically it is desired that the film adhere well without cracks or delamination.

In contrast, within the nanomembrane platform, stress actuation has been translated into a way to fold nm-thickness films into micro- and nanomechanical objects. ${ }^{7}$ A simple example is a bilayer with one layer intentionally deposited with more residual stress than the lower layer. When this bilayer film is released from the holding substrate (the central feature of the nanomembrane technology), the intrinsic stress gradient established in the bilayer material during deposition drives the defined film to curl in a predictable fashion. Uniform compressive strain in a single-crystalline thin film has been achieved by epitaxial growth on a substrate with a smaller lattice constant or vice versa for tensile strain. A membrane that is uniformly compressively strained relaxes by lateral expansion when it is released from the holding substrate, and the opposite for a tensilely strained singlelayer membrane. A bilayer with different strains in the two layers will change its shape to relax the strain when it is released from the holding substrate. Formation of wrinkle-like structures occurs upon partial release of a compressively strained film, as

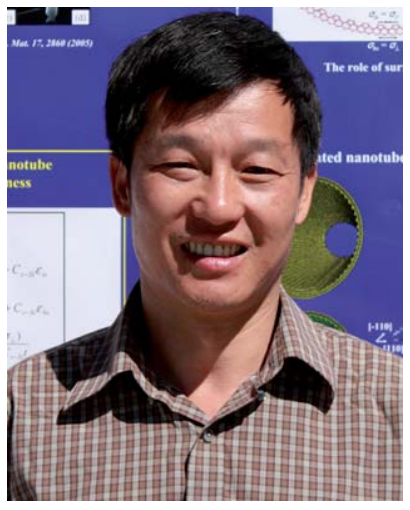

Feng Liu
Feng Liu is a professor in the Department of Materials Science and Engineering at the University of Utah. His research interests lie in materials modeling and simulation from the atomic to mesoscopic scales, with a recent focus on properties of surfaces, interfaces and nanostructures, mechanisms of growth of thin films and of selfassembly of nanostructures, and nanomechanical architectures of nanomembranes. Liu received his Ph.D. degree in Chemical Physics from Virginia Commonwealth University and was a postdoctoral research fellow at Rutgers University and Oak Ridge National Lab. He has authored 140 publications and holds 4 patents. He received the Humboldt Senior Research Award in 2008.

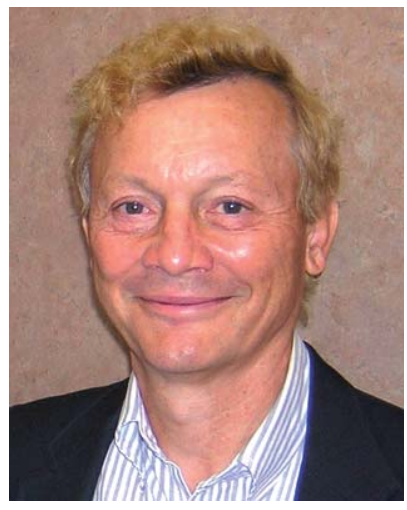

Max G. Lagally
Max G. Lagally is the Erwin W. Mueller Professor of Materials Science and Physics at the University of Wisconsin-Madison. His research focuses on growth and structure-properties relationships of materials at the nanoscale, primarily semiconductors. He received his B.S. degree from the Pennsylvania State University and his Ph.D. degree from Wisconsin, both in physics. He has been a Humboldt Fellow at the Forschungszentrum, Jülich, Germany. He is a member of the U.S. National Academy of Engineering and the German National Academy of Sciences - Leopoldina, and has received numerous other awards. He has founded two companies, nPoint, Inc. and SonoPlot, Inc. 
the released regions expand to relax strain. A trilayer membrane in which the top and bottom layers are balanced will, like a single layer, expand or contract, depending on the sign of the strain.

Extensive work has been done since 2000 to achieve control of these phenomena. These efforts succeeded in transforming a trial and error process into a nanofabrication technique for reliable and parallel mass production of identical mixed-dimension nano- and microstructures. A variety of nanomechanical architectural designs has been realized with various materials, including semiconductors, ${ }^{7-11}$ metals, ${ }^{25}$ organic materials, ${ }^{26}$ and insulators, ${ }^{27}$ as well as combinations of them. ${ }^{12,13,27}$

Because strain relaxation is the driving force for bending 2D strained sheets or ribbons into 3D structures, the design principles for fabrication of different classes of $3 \mathrm{D}$ nanoarchitectures can be described based on total strain energy minimization within the elastic regime. Different nanoshapes can be designed using the geometric and elastic properties of membranes and the magnitude of the gradient of built-in strain. ${ }^{11,28,29}$ A freestanding strained membrane attached at one end can bend or wrinkle, depending on magnitude of strain and elastic properties of the material. In the bending regime it is possible to tune the radius of curvature by varying the amplitude of the strain gradient and the film thickness. Similarly, in the wrinkling regime the amplitude of wrinkled structures and the spacing between adjacent wrinkles depend on intrinsic parameters, such as the intensity of the strain field and film thickness. Continuum elasticity theory is used to establish generic design principles (i.e., universal physical conditions and geometric relationships) that control the size of nanostructures in a single class, as well as the formation of different classes of nanostructures. ${ }^{1129-37}$ Definition of extrinsic parameters, such as the geometry of the film before release, the alignment of the starting edge for the etching process in a certain crystallographic direction, and the etching rate of the sacrificial layer can be used to design the desired ultimate 3D structure.

All-semiconductor membranes with thicknesses ranging from a few to hundreds of nanometers and the proper strain gradients roll up in tubular structures with diameters scalable from tens of micrometers down to $10 \mathrm{~nm}$. Rolled-up tubes are robust and stable. High-resolution transmission electron microscopy and $\mathrm{X}$-ray diffraction reveal that single-crystalline nanomembranes preserve their crystal structure after release and rollup. ${ }^{38,39} \mathrm{X}$-ray diffraction has also been used to probe deformation of the crystal lattice in the bent membrane. ${ }^{39}$ Closed tubes can withstand mechanical stimulation applied by a sharp probe, ${ }^{40}$ and they remain closed and do not open when fluids flow through. ${ }^{\mathbf{1 8 4 1}}$ They survive after conventional microfabrication procedures, e.g., photolithography and reactive ion etching. ${ }^{41}$ The mechanical strength of rolled-up membranes can be increased by fabricating multi-walled tubes, which, like a rug rolling up, are obtained simply by increasing the pattern length and the release length with a longer etching time. Thin Group-IV based membranes fabricated in the form of rolled-up tubes show high thermal stability. ${ }^{\mathbf{1 7 , 4 2 , 4 3}}$ The tubes do not collapse for annealing at temperatures at least as high as $800{ }^{\circ} \mathrm{C} .{ }^{\mathbf{1 7}}$

Many quite beautiful structures can be made by combining top-down with bottom-up processing. But are they useful? It has been said that "Sometimes the results of an experiment are so beautiful that researchers assume they must be useful, too". ${ }^{37}$ Detailed investigation of mechanical, optical, and electronic properties of nanomechanical architectures has unveiled their unique properties and their full potential as building blocks for highly integrative systems. High thermomechanical stability, flexibility, and circular and hollow geometries are among the most interesting features. The addition of metals, insulators, or organics has allowed fabrication of hybrid structures, for example, alternating materials with different properties arranged in a radial geometry.

The approaches reviewed in this paper include 1) accurate design of the desired nanostructure (i.e., material, geometry, size, and position on the holding substrate), 2) definition and fabrication of $2 \mathrm{D}$ templates according to the established design, and 3) release of the thin film into a 3D or quasi-3D object. We also describe several properties and proposed applications of nanomechanical architectures, with the goal of stimulating interest in the topic of nanomembranes. In the next section we focus on the continuum elasticity models used to establish design parameters for the fabrication of different classes of nanomechanical architectures. Sections 3 and 4 focus on fabrication and characterization of the fabricated membranes, respectively. Section 5 describes several potential uses of rolled-up or wrinkled structures to implement simple and hybrid functionalities. Section 6 provides conclusions and an outlook.

\section{Generic design principles}

The nanostructures described in this review are created by templated 3D fabrication and self-assembly based on the principle of stress-driven actuation. In the last decade, a very high degree of control of the residual stress (strain) in a film has been achieved and the stress actuation method has been developed as a way to shape nm-thick films into tubes, spirals, rolled-up "rugs", and periodic wrinkles. The fabrication process consists of two parts. In the first part, the materials system and geometry, size, and position are defined on a $2 \mathrm{D}$ template layer by using standard deposition and patterning techniques. In the second part the membranes are released from the substrate, with the consequent release of the stored stress (strain) initiating the bending or rolling process, so that the $2 \mathrm{D}$ membrane assumes the intended final 3D shape.

In this section we review the generic design principles relevant to the nanomechanical architecture of strained nanomembranes. We focus on three aspects: 1) selection of the 3D geometry, 2) size scalability, and 3) positioning 3D nanostructures on the substrate.

\subsection{Designing nanoarchitectures with various geometries}

Because strain relaxation is the driving force for bending $2 \mathrm{D}$ strained membranes into 3D structures, the design principles for fabricating different classes of 3D nanoarchitectures can be described by total strain energy minimization within the elastic regime. ${ }^{11,31-34,44}$ Different nanoshapes can be designed using the elastic and geometric properties of membranes and the magnitude of the built-in strain gradient in them.

We first describe the influences of elastic properties on the final rolled-up nanoshape. We consider both materials with isotropic and with anisotropic properties. Isotropic elastic properties are defined as ones that are identical in all directions, while 
anisotropic ones are direction dependent. For instance, singlecrystal $\mathrm{Si}, \mathrm{Ge}$, and $\mathrm{SiGe}$ are elastically anisotropic materials. The Young's modulus of $\mathrm{Si}$ has the smallest value along the $<100>$ directions, ${ }^{45-47}$ i.e., $<100>$ is the mechanically softest direction. Intuitively, membranes with anisotropic elastic properties will curl along the softest direction. Energetically it is therefore favored for the anisotropic $\mathrm{Si} / \mathrm{Ge}$ strained membrane to curl only along the $<100>$ soft direction, because curling occurs along the direction with the minimum bending energy, i.e., the most compliant direction.

For such materials with elastic anisotropies, the final rolled-up shape now depends critically on the alignment between the desired curling direction and the most compliant direction. When the desired curling direction is aligned with $\langle 100\rangle$, the strained membrane curls along $<100>$ to form a tube or ring; if the desired curling direction is aligned off $<100>$ (i.e., a strip is patterned with its long direction NOT along $<100>$ ), the strained membrane still curls along $\langle 100\rangle$, forming a coil. The elastic anisotropy dominates in the formation of nanotubes and nanocoils. This design principle has been demonstrated experimentally. ${ }^{11,48}$ As shown in the upper panel of Fig. 1, the strained membrane is patterned into cantilevers along two different directions: one along $<100>$ (the most compliant in $\mathrm{Si} / \mathrm{Ge}$ ) and the other along $<110\rangle, 45^{\circ}$ off the $\left.<100\right\rangle$ direction. ${ }^{11}$ The lower panel of Fig. 1 shows that $\mathrm{Si} / \mathrm{Ge}$ strained bilayer membranes patterned into $<100>$-oriented cantilevers curl along the most compliant $<100\rangle$ direction, forming rings. In contrast, $<110>$-oriented cantilevers, also curling along $<100>$, form coils. The detailed experiment is described in ref. 11 .

Another important variable is the relative thicknesses of the two layers making up a bilayer strained membrane. In general, the strain in a bilayer strained membrane can not only relax elastically via bending but also can relax via stretching of the whole film when it is freestanding, depending on the thickness ratio of the two layers. ${ }^{31}$ The influence of the membrane

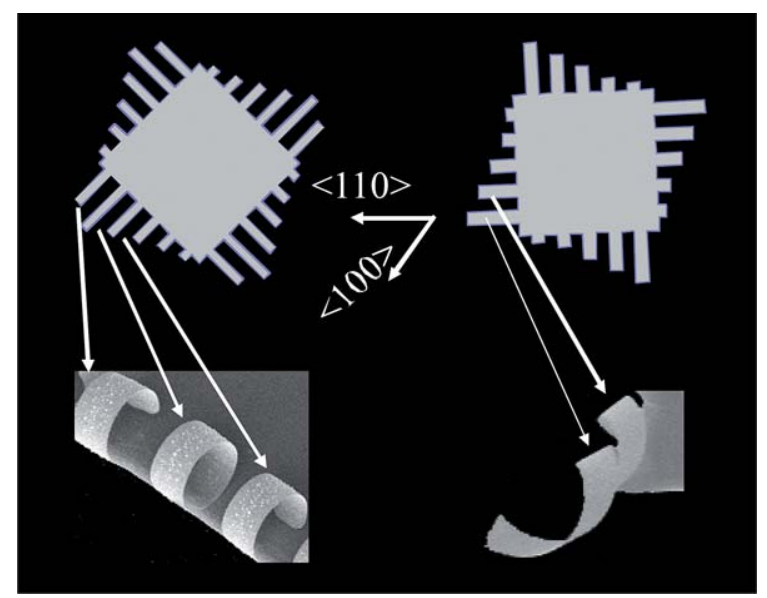

Fig. $1 \mathrm{Si} / \mathrm{SiGe}$ bilayer cantilever membrane released from patterned substrates. $<100>$-oriented cantilevers roll into nanorings (left images), while $<110>$-oriented cantilevers curl into nanocoils (right images), as they prefer to fold along the most compliant $<100>$ direction. The nanorings have a thickness of $60 \mathrm{~nm}$, radius of $\sim 3.2 \mu \mathrm{m}$, and width of $3 \mu \mathrm{m}$; the nanocoils have a thickness of $76 \mathrm{~nm}$, radius of $\sim 2.8 \mu \mathrm{m}$, and width of $4 \mu \mathrm{m}$. Reprinted from ref. 11 with permission. thickness on bending or stretching of a bilayer can be described as follows. When a compressively strained layer grows on a substrate, its lattice relaxes outward, which applies both a force (stress) and a torque (moment) to the substrate, the former tending to stretch the substrate uniformly and the latter to bend the substrate downward. The magnitude of the force is proportional to the strain and the film thickness $(F \sim \varepsilon d)$; the magnitude of torque equals the force $(F)$ times the distance $(l)$ from the mean position of the applied force (middle of the film) to the line of the center of the whole system [middle of the (film + substrate)], as illustrated in Fig. 2. For a thickness much less than that of the substrate, the film applies a relatively small force but a large torque because of a large $l$, as shown in Fig. 2(a). Consequently, the film bends the substrate without much stretching. The bending increases with increasing film thickness, following the classical Stoney formula, ${ }^{44}$ but is typically hardly noticeable because the film is generally very thin compared to the substrate. Conversely, for a thickness much greater than that of substrate, the film applies a relatively large force but a small torque because of a very small $l$, as shown in Fig. 2(c). Consequently, the film stretches the substrate uniformly without much bending. The bending decreases with increasing film thickness, because the torque decreases as force is applied more and more closely to the center of the whole system (diminishing $l$ ). When both thicknesses are comparable, the film bends as well as stretches the substrate, as shown in Fig. 2(b). The bending behavior now follows the classical Timoshenko formula. ${ }^{34}$

When the elastic properties are isotropic, the bending, rolling, or curling behavior is not so intuitive. It is, however, a situation that can be treated quantitatively. Geometric conditions and shear stress now affect the final membrane morphology. Fig. 3 shows the conditions schematically. If the strained membrane has isotropic elastic properties, it tends to roll into a tube or rug when it is wide or a ring when it is narrow. A long narrow strip may, however, form a coil, as described below, and involves minimization of energy that involves a shear term.

The characteristic bending curvature $(\kappa)$ can be calculated using the Timoshenko formula, shown in eqn (1). ${ }^{31-34}$ For simplicity, we assume the two layers have the same thickness $(t / 2)$ and the same isotropic elastic constants. Then eqn (1) simplifies to $\kappa=-\left(3 \varepsilon_{m}\right) /(2 t)$. The bilayer membrane will roll into a tube or ring whose rotations have a characteristic radius $R_{0}=1 / \kappa$ and a perimeter length $L_{0}=2 \pi R_{0}$.

$$
\kappa=-\frac{6 E_{1} \varepsilon_{m} t_{1}}{E_{2} t^{2}} \gamma
$$

with

$$
\gamma=\frac{1+\beta}{1+4 \alpha \beta+6 \alpha \beta^{2}+4 \alpha \beta^{3}+\alpha^{2} \beta^{4}},
$$

where $\alpha=E_{1} / E_{2}, E_{1}$ and $E_{2}$ are respectively the biaxial Young's moduli of layers 1 and $2, \varepsilon_{m}$ is the misfit strain between these layers, $\beta=t_{1} / t_{2}$ is the ratio of layer 1 thickness $t_{1}$ and layer 2 thickness $t_{2}$, and $t=t_{1}+t_{2}$ is the total thickness of the bilayer membrane.

As the material is elastically isotropic, the membrane is able to curl or roll along all directions that have equal bending energy. But there is also an optimum tube radius, as shown above. When a strained membrane is longer than the perimeter length $\left(L>L_{0}\right)$, 
(a)

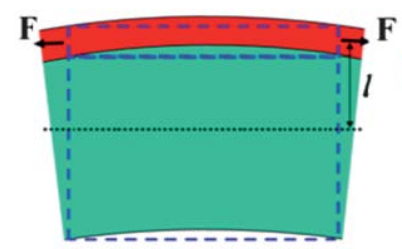

(b)

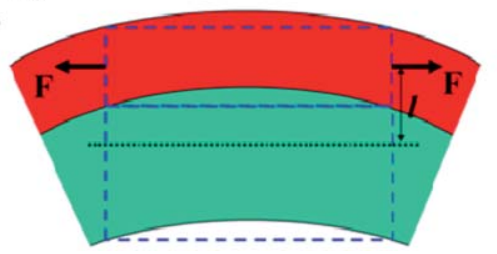

(c)

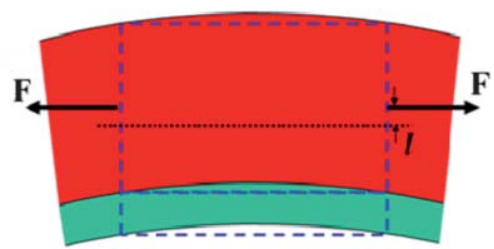

Fig. 2 2D schematic illustration of the force $(F)$ and torque $(F \times l)$ applied by a compressively strained film (red) grown on a substrate (green) for different film-to-substrate thickness ratios. Reprinted from ref. 31 with permission.

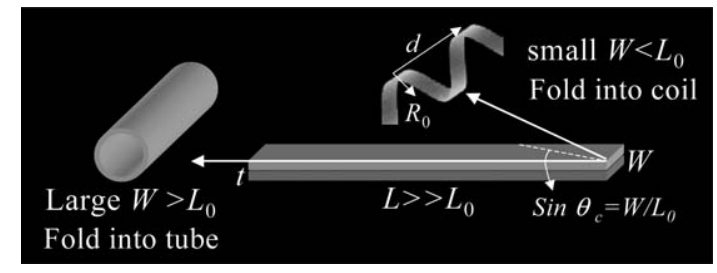

Fig. 3 Schematic diagram of rolling or curling a strained bilayer membrane upon release, illustrating the geometric parameters to determine the membrane morphology. The arrows indicate the folding direction. Reprinted from ref. 11 with permission.

some extra energy may be associated with it. Any tube that forms can adopt the optimum radius $R_{0}$ with minimum bending energy only for the first revolution, after which it must adopt a larger radius, thus failing to minimize the total energy. A wide membrane $\left(W>L_{0}\right)$ that is being underetched still has no choice but to roll into a tube. There exists a geometric condition to form coils, i.e., $W<L_{0}$. The strained membrane can roll into a coil by choosing a curling direction having an angle $\theta$ with its long edge, so that all the rotations in the coil adopt the optimal radius $R_{0}$ with the minimum bending energy, but at some cost in shear energy. In contrast, if the membrane rolls into a ring with multiple rotations by curling along its long edge, only its first rotation can adopt $R_{0}$, while additional rotations must adopt a radius larger than $R_{0}$, with extra cost of bending energy. From the energy standpoint, a coil can always be favored to form over a tube when $W<L_{0}$. There is a critical angle $\theta_{c}=\sin ^{-1}\left(W / L_{0}\right)$ to ensure the membrane rolls into a coil with a certain pitch $(d)$, as shown in Fig. 3. If $\theta<\theta_{c}$, the membrane rolls into a tube with a continuous tube wall, i.e., "a coil with zero $d$ ". Therefore, there exist two required geometric conditions for coil formation, $W<L_{0}$, and $\theta>\theta_{c}$.

The total elastic energy for tube- $v s$-coil formation can be calculated, in terms of length, width, and thickness of the starting membrane. Fig. 4 shows a phase diagram for a $60 \mathrm{~nm}$ thick $\mathrm{SiGe} / \mathrm{Si}$ bilayer membrane, by assuming it has isotropic elastic properties. It defines the geometric regime for tube- $v s$-coil formation. For a given film length $L<L_{0}$, tubes dominate no matter how wide the bilayer membrane is, i.e., the minimum length for coil formation is $L_{0}$. Once $L>L_{0}$, there exists a critical membrane width above which tubes form and below which coils form. For example, when $L / L_{0}=10, W / L_{0}$ has to be smaller than 0.01 for coil formation; above this value, tube formation is favored. Conversely, for a given membrane width $W$, there exists a critical length for coil formation $L_{0}$. Wider membranes (larger $W / L_{0}$ ) would require longer membranes (larger $L / L_{0}$ ) for coil formation. For example, when $W / L_{0}=0.02, L / L_{0}$ has to be larger than 100 for coil formation; below this value, only tubes form. There exists an upper limit of width, $W$, for coil formation, $3 \%$ of $L$, as shown in Fig. 4 . The phase boundary also depends on the magnitude of membrane elastic properties. Increasing bending modulus or decreasing shear modulus suppresses tube formation and favors coil formation, so that the boundary line in Fig. 4 shifts upward either with increasing bending modulus or decreasing shear modulus.

As mentioned at the start of this section, when elastic anisotropy is introduced, the strained membrane curls only along the most compliant direction, in other words, that factor dominates the energy. The formation of coils is determined by the alignment of the curling direction and the soft direction. The helicity angle, chirality, diameter, and pitch of coils are all width dependent, as shown in Fig. 2 in ref. 48.

A bilayer strained membrane can also wrinkle when released from the holding substrate..$^{29,49-53}$ In general, wrinkles are caused by the interplay between bending and stretching in the presence of geometric constraints. Fig. 5(a) shows a 3D partially released bilayer strained membrane $(L \gg h)$, the released portion of which is free to relax elastically. ${ }^{29}$ There is a constraint, i.e., the boundary condition that no elastic strain relaxation can occur where the membrane is still bonded to the substrate, as shown by the dashed line. The strain gradient and the average strain are

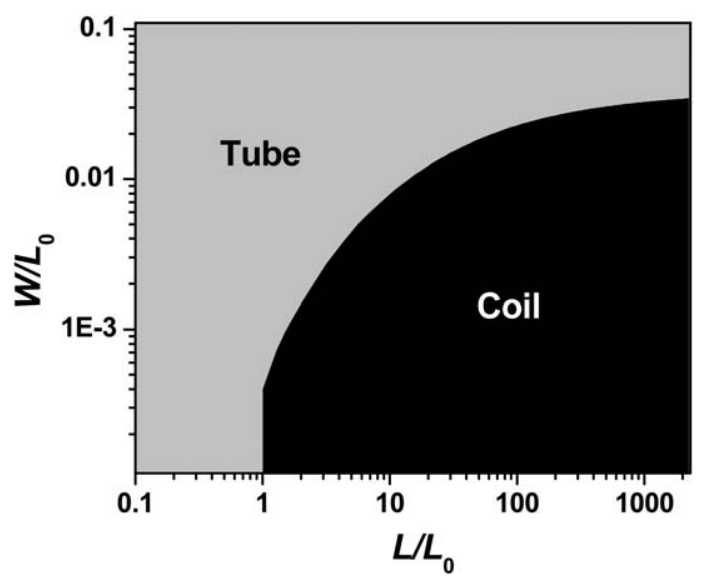

Fig. 4 Log-log plot of thermodynamic stability for nanotube or nanocoil formation, as a function of reduced film length $L / L_{0}$ and reduced width $W / L_{0}$, for a given set of bending and shear moduli. Here the $\mathrm{SiGe} / \mathrm{Si}$ bilayer membrane is treated as an isotropic material. Reprinted from ref. 11 with permission. 
defined as $\Delta \varepsilon=\varepsilon_{1}-\varepsilon_{2}$ and $\bar{\varepsilon}=\left(\varepsilon_{1} t_{1}+\varepsilon_{2} t_{2}\right) /\left(t_{1}+t_{2}\right)$, respectively, in a bilayer strained membrane.

We first consider wrinkle formation in elastically anisotropic materials. Again, the membrane will deform only along the most compliant direction. As shown in Fig. 5, if the y direction is the most compliant direction, the bilayer strained membrane will bend and/or stretch freely in the y direction, forming a tube. If the $\mathrm{x}$ direction is the most compliant direction, the sheet will attempt to bend and stretch in the $\mathrm{x}$ direction, but the constraint causes the formation of wrinkles. This qualitative explanation is valid for single-layer or multilayer membranes. Wrinkling has been demonstrated experimentally, ${ }^{49}$ as shown in Fig. 6 , in an end-constrained single $\mathrm{SiGe}$ freestanding layer after release from the host substrate along a trench that is mechanically scratched in the $<100>$ direction, the most compliant direction in SiGe. Three groups of SiGe samples with different Ge composition and thickness were prepared. They all show wrinkles forming in $<100>$ direction after release. The wavelength of wrinkling is measured via atomic force microscopy. The detailed experiment is described in ref. 49 .

Fig. 7 shows that the measured wavelength of wrinkling starts at a certain lateral etching length $h$ for all three groups of samples and then increases with increasing $h$, (as defined in Fig. 5), indicating there is a critical $h_{c}$ for wrinkle formation. Below $h_{c}$, the geometric constraint does not allow strain to relax along the trench direction, even though it is the most compliant direction. Energetically, strain could less likely relax along the transverse direction, i.e., the hard $\langle 110\rangle$ direction, so it will be retained mostly in the membrane until $h>h_{c}$. Wrinkles can only form when $h>h_{c}$. The wavelength and amplitude of wrinkles as a function of the strain gradient has also been investigated (Fig. 2 in ref. 49). As the strain gradient increases, the wavelength decreases while the amplitude increases. Because the increased strain relaxes preferentially in $<100>$ directions, narrow and high wrinkles form.

We now consider strained bilayer membranes with isotropic elastic properties. The strained membrane is able to wrinkle along all directions that have equal bending/stretching energy, if no boundary condition exists. What it does depends on the magnitude of the strain gradient created in it. Qualitatively, if the strain gradient is large, the strained layer must be much thinner than the unstrained layer before releasing the bilayer, to prevent plastic deformation or relaxation and so a large bending moment

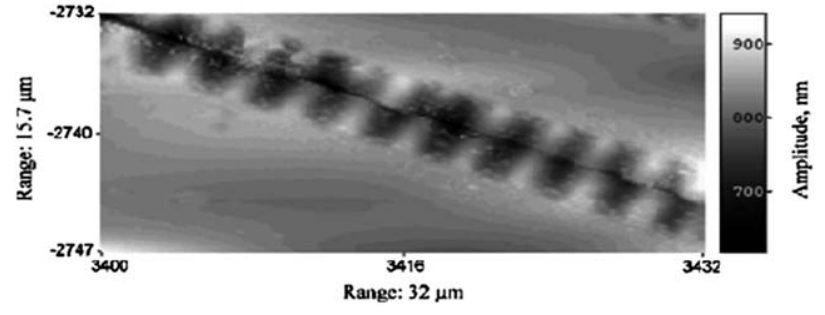

Fig. 6 Atomic force microscopy image of a wrinkled freestanding $\mathrm{Si}_{0.7} \mathrm{Ge}_{0.3}$ layer. The wrinkles form at both the edges and along the elastically soft $<100>$ directions. Wrinkles are shown as bright regions separated by dark regions. Reprinted from ref. 49 with permission.

exists, as shown in Fig. 2(a), which will bend or curl the membrane. Conversely, if the strain gradient is small, the strained layer can be thicker than the unstrained layer, so a large force exists to stretch the bilayer, as shown in Fig. 2(c), leading to wrinkle formation if appropriate boundary conditions exist.

For isotropic elastic properties, again the geometric condition is important for deciding the formation of tubes $v s$. wrinkles. The etching length $(h)$ determines the released region of the bilayer strained membrane (see Fig. 5). When $h$ is small $(L \gg h)$, the geometric confinement in the $\mathrm{x}$ direction limits relaxation in $\mathrm{x}$; instead strain is relaxed via bending and stretching in the $y$ direction only (unlike the anisotropic case). Increasing $h$ reduces the effect of the constraint in $\mathrm{x}$ (it moves the constraint some distance away), and strain can now begin to be relaxed via bending and stretching in both directions. There is no geometric constraint for strain relaxation in the y direction (free boundary condition), while there is a geometric constraint for strain relaxation in $\mathrm{x}$ direction (fixed boundary condition). Stretching and/or bending with a constraint leads to wrinkling. ${ }^{29,49-53}$ There exists a critical etching length for wrinkling, $h_{c}$. The geometric condition to determine wrinkle formation is $h>h_{c}$. Large $h$ should allow the bilayer to roll better if the strain gradient is large. A small strain gradient does not provide enough bending moment to roll. If the film cannot roll, it will stretch at the free end, leading to wrinkling (stretching with a constraint) if $h$ is large enough.

The total elastic energy of bent and wrinkled structures has been calculated, in terms of strain gradient, etching length, and layer thickness, for both bilayer ${ }^{11,31-34}$ and single-layer ${ }^{29,49,53}$
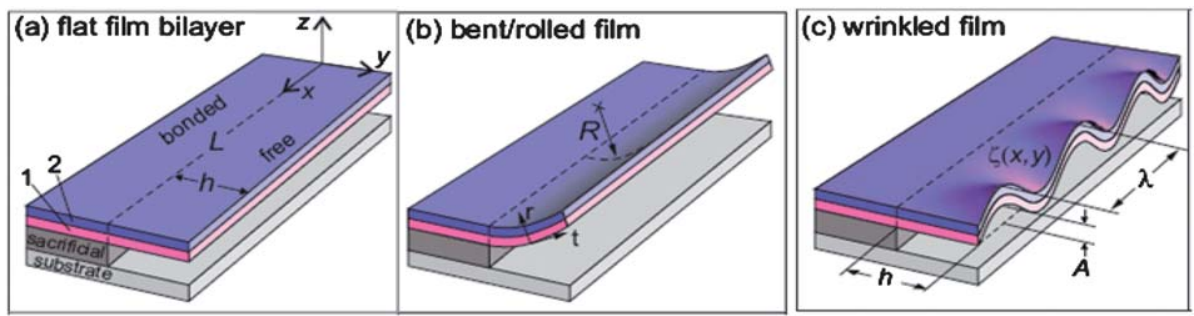

Fig. 5 Schematic diagrams of distortions of a strained bilayer membrane with isotropic elastic properties upon release, illustrating the geometric parameters to determine the membrane morphology. (a) Free-hanging bilayer film comprising two layers with thicknesses $t_{1}$ and $t_{2}$, subject to biaxial strains $\varepsilon_{1}$ and $\varepsilon_{2}$, respectively. The total length of the bilayer is $L . h$ is the etching length, defined as the distance over which the release layer has been etched away. (b) A bent film with inner radius $R$, and (c) wrinkled structure with deflection profile $\zeta(x, y)$, amplitude $A$, and wavelength $\lambda$. Reprinted from ref. 29 with permission. 


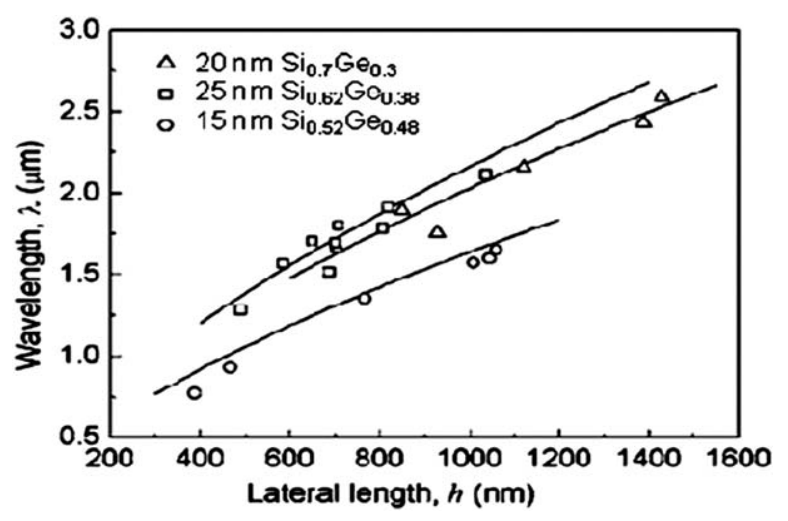

Fig. 7 Measured wavelength with respect to the lateral length $h$, as defined in Fig. 5, for three groups of samples. The solid lines represent the calculated wavelength, Reprinted from ref. 49 with permission.

strained membranes. In this calculation, the membranes are assumed to have isotropic linear elastic properties. The condition to determine bending and wrinkling can be calculated as a function of these variables. For example, Fig. 8 shows a calculated phase diagram ${ }^{29}$ for a $20 \mathrm{~nm}$-thick $\mathrm{In}_{0.1} \mathrm{Ga}_{0.9} \mathrm{As} /$ GaAs bilayer. For small $\Delta \varepsilon=0.20 \%$ and $\bar{\varepsilon}=0.36 \%, h_{0}$ is calculated as $\sim 700 \mathrm{~nm}$. The lower-left part of Fig. 8 shows that when $h<h_{0}$, bending is dominant; when $h>h_{0}$, wrinkling starts to dominate. To shift bending to wrinkling, one can increase $\bar{\varepsilon}$. Fig. 8 shows that by increasing $\bar{\varepsilon}$ (the dashed line shows $1 \%$ tensile strain), the phase boundary shifts upward and wrinkling becomes more dominant (the wrinkling region is enlarged). This can be understood as follows. Large $\bar{\varepsilon}$ can be achieved by either increasing the strained-layer thickness $d_{2}$ or decreasing the unstrained-layer thickness $d_{l}$ by assuming $\Delta \varepsilon$ is constant. Doing so will make wrinkling (stretching with constraints) energetically favorable without much bending, as shown in Fig. 5(c). The color bars in Fig. 8 show that in the bending regime, the inner radius of tubes decreases with increasing $\Delta \varepsilon$, while in the wrinkling regime, the wavelength of wrinkles increases with increasing $h$.

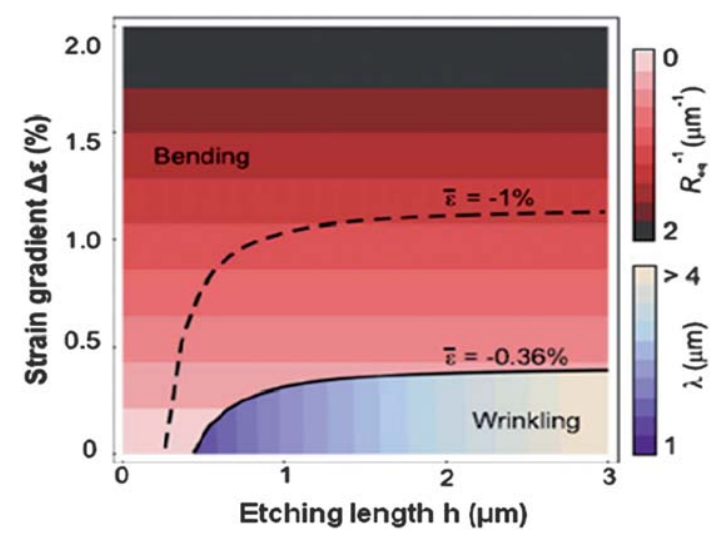

Fig. 8 Phase diagram of favorable film shapes based on an energetics comparison between bent and wrinkled structures. $R_{\text {eq }}$ is the inner radius of the bent structure and $\lambda$ is the wavelength of the wrinkled structure. Reprinted from ref. 29 with permission.

\subsection{Scalability of nanomechanical architectures}

A century ago, the bending of a bimetallic strip was analyzed by Timoshenko ${ }^{34}$ with continuum elasticity theory. The same formula has been applied to model many other materials systems, such as epitaxially grown bilayer thin films. ${ }^{11,31-34}$ Our design principles for nanomechanical architectures of strained bilayer membranes, strain sharing multilayer membranes, and even membranes made from single materials with a strain gradient, are therefore based on the classical Timoshenko formula ${ }^{34}$ However, as the strained membrane gets sufficiently thin, stresses associated with the existence of nearby surfaces become important in the bending, and the classical Timoshenko formula must be modified to account for them..$^{30,35,36,54,55} \mathrm{We}$ discuss these modifications below.

2.2.1. Scaling the diameter of micro- and nanotubes. The general expression for the bending curvature $(\kappa)$ of a bilayer stained membrane based on the Timoshenko formula ${ }^{11,31-34}$ is given as eqn (1). Eqn (1) indicates that $\kappa$ is dependent on the misfit strain and the thickness and elastic constants of the bilayer membrane. The nanotube radius is related to the bending curvature of a given strained-bilayer membrane. The diameter of rolled-up nanotubes (RUNTs) can therefore be scaled by varying the misfit strain $\left(\varepsilon_{m}\right)$, the ratio of elastic constants $(\alpha)$, and the ratio of membrane thicknesses $(\beta)$. The scaling of diameters of RUNTs made from initially strained multi-material, multilayer membranes can also be modeled by eqn (1). For example, the diameters of $\mathrm{SiGe} / \mathrm{Si} / \mathrm{Cr}$ and $\mathrm{SiGe} / \mathrm{Si} / \mathrm{Si}_{\mathrm{x}} \mathrm{N}_{\mathrm{y}} / \mathrm{Cr}$ microtubes have been estimated this way. ${ }^{56}$

Because of the large surface-to-volume ratio, stresses associated with the presence of surfaces are expected to play an important role in determining the bending of nanomembranes. As the membrane gets thinner, the influence of surface related stresses becomes more dominant. The classical Timoshenko formula neglects surface and interface stresses and is therefore valid, in principle, only for relatively thick films for which the surface/interface and the atomic structure can be neglected. As shown in Fig. 9, there exist significant discrepancies between Timoshenko's formula (dashed line) and experimental results (square dots) for very thin membranes.

Because the surface of most materials is relaxed in some way, either via reconstruction or via chemical modification, one can think of the surface region as a separate phase with its own elastic properties. These are negligible when the nanomembrane is thick, but become important when it is very thin. The properties of this separate phase must be included in the Timoshenko formula. Bending induces an additional surface stress, which equals the product of bending strain and the surface layer elastic constant, namely "surface stress induced by large bending". ${ }^{35}$

The Timoshenko formula can be modified to include surface effects. The detailed derivation is described in ref. 35. As an example, Fig. 9(a) plots the diameters of a series of GaAs/InAs nanotubes made with diameters ranging from 20 to $500 \mathrm{~nm}$ by varying the thickness of the GaAs layer..$^{57}$ The dashed line shows smaller diameters predicted by the classical Timoshenko formula. For a GaAs/InAs strained bilayer, the upward bending is induced by the relaxation of misfit strain. When the bilayer is very thin, the surface stress can influence the bending behavior. 
(a)

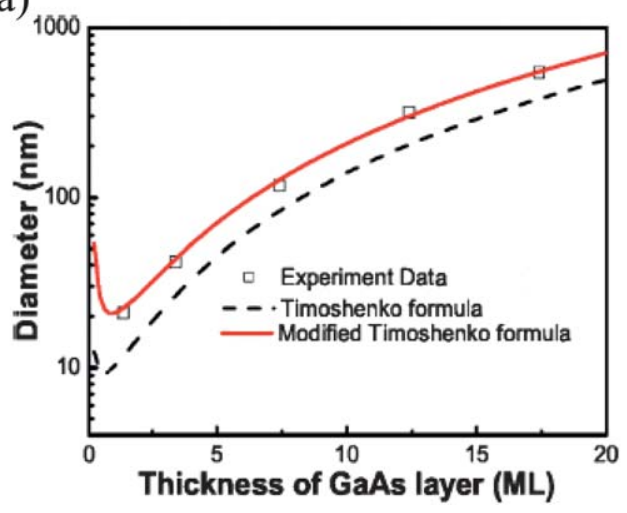

(b)

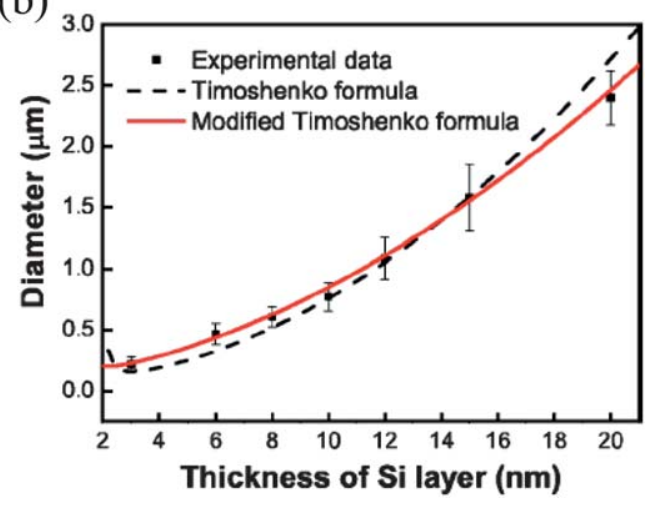

Fig. 9 Comparison of results with the classical and modified Timoshenko formulas. (a) Diameters of GaAs/InAs nanotubes as a function of thickness of the GaAs layer with the thickness of the InAs layer fixed at two monolayers (MLs). See ref. 57 for experimental details. (b) Diameters of pure Si microand nanotubes as a function of thickness of the Si layer with the thickness of the strained Si layer fixed at $2 \mathrm{~nm}$. See ref. 58 for experimental details. Reprinted from ref. 35 with permission.

The intrinsic surface stress of GaAs is, by nature, different from that of InAs due to the surface reconstruction. The differential intrinsic surface stresses between top GaAs and bottom InAs surfaces causes an effect opposite to the bending due to relaxation of misfit strain, causing the radius of curvature to increase. Modifying the classical Timoshenko formula by adding this effect produces a good fit to the data.

The same design principle can be used to scale the diameters of RUNTs made from a single-material membrane. Singlematerial membranes can, for example, be a membrane of $\mathrm{Si}$ in which part of the thickness is strained. This structure can be achieved by growing $\mathrm{Si}$ on a Ge layer at a low temperature (300 ${ }^{\circ}$ C) via molecular beam epitaxy. ${ }^{58}$ The initial Si layers are strained (although partially relaxed and thus with dislocations), but then begin to relax plastically more fully with increasing film thickness, leading to a strain gradient in the single layer. The detailed fabrication is described in Sec. 3.1. For rolling up single-material strained membranes, $\alpha=1$ and eqn (1) reduces to a simpler form

$$
\kappa=-\frac{6 \varepsilon_{m} t_{1}}{t^{2}}(1+\beta)^{-1}
$$

The diameter of a pure-Si RUNT is estimated by using eqn (3) and compared to experiment, as shown in Fig. 9(b). The classical Timoshenko formula (dashed line) does not fit the data well. Here the surfaces phases on both sides of the nanomembrane are the same and the differential surface stress mentioned above can play no role. But, because the radii of curvature of the inner and outer surfaces are different, the strain induced in the surface phases on each side differs. By adding this effect into eqn (3), the calculation fits the data well.

2.2.2 Bending induced by unbalanced surface stresses. As mentioned above, surface reconstruction causes an intrinsic surface stress. ${ }^{36}$ For example, the clean $\mathrm{Si}(001)$ surface exhibits a $(2 \times 1)$-type reconstruction, as shown in Fig. 10(a). Because of the tetrahedral bonding of $\mathrm{Si}$ atoms, the surface dimers on one atomic layer rotate by 90 degree in adjacent atomic layers. For a membrane with an even number of atomic layers, the dimers on the top and bottom surface are parallel to each other, as shown in Fig. 10(b) and 10(c) for a six-atomic-layer membrane. For a membrane with an odd number of atomic layers, the dimers are perpendicular to each other, as shown in Fig. 10(d) for a fiveatomic-layer membrane. This odd-even alternation of surface dimer orientations will have a direct impact on the bending of $\mathrm{Si}$ nanomembranes. In an even-layer membrane, the intrinsic stresses on the top and bottom surfaces cancel each other [Fig. 10(b) and 10(c)]; while in an odd-layer membrane, there exists a surface-stress imbalance between its top and bottom surfaces [Fig. 10(d)], creating a unique self-driving force to bend the membrane. Atomistic simulations have been performed to study how the intrinsic surface stress affects the bending of ultrathin membranes. ${ }^{36}$ It has so far not been possible to make membranes this thin or ones that are freestanding and clean on both sides.

Surface stress caused by reconstruction of the clean surface will act with or against the misfit strain in bending SiGe bilayer membranes, depending on the orientation of surface dimers. Normally, a SiGe membrane bends always towards the Si side, as illustrated in Fig. 10(e). Fig. 10(f) shows surface stress acting with misfit strain to increase the bending toward the Si side, as seen by comparing Fig. 10(e) to Fig. 10(f). However, if we switch the top and bottom surface dimer orientation, as shown in Fig. 10(g), the surface stress will act against misfit strain to decrease the upward bending. For a thin enough membrane or weak lattice mismatch strain the surface stress can be strong enough to reverse the "normal" bending direction defined by misfit strain, making the film bend downward, toward the Ge side.

\subsection{Accurate positioning of nanomechanical structures}

The position in the surface plane of nanomechanical structures is defined by patterning the corresponding $2 \mathrm{D}$ strained membranes via lithographic techniques. Two most common techniques are photo- and electron-beam (e-beam) lithography. Conventional 
(a)

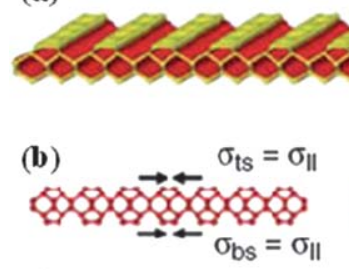

(d)

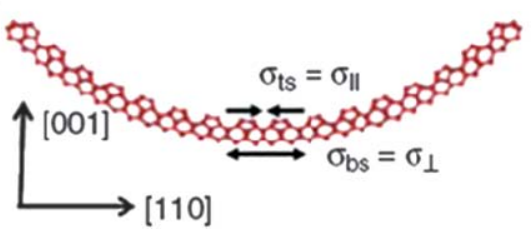

(e)
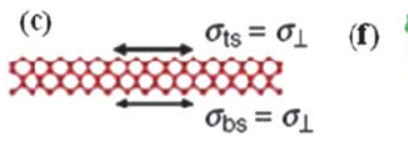

"⿻
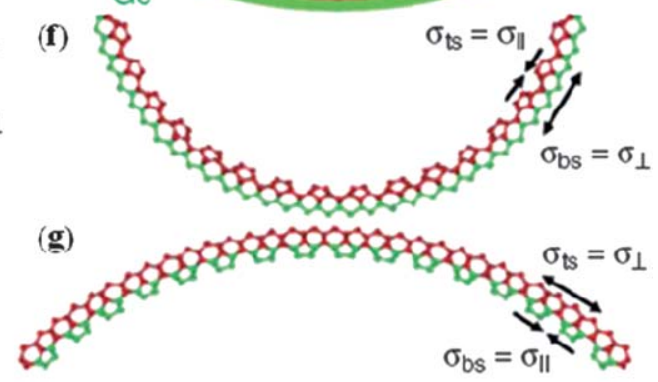

Fig. 10 Bending of a nanomembrane induced by surface reconstruction. (a) Schematic diagram of the $\operatorname{Si}(001)-(2 \times 1)$ surface reconstruction, consisting of rows of dimers, which introduce a large surface stress anisotropy. (b)(c) Side views of a six-layer Si film, showing the parallel surface dimer orientations and the balancing surface stress with the dimer bonds on both the top and bottom surfaces. (d) Side view of a five-layer Si film, showing the orthogonal surface dimer orientations and the simulated self bending induced by surface stress imbalance. (e) Conventional picture of a SiGe bilayer film that always bends toward the Si side. (f) Side view of a simulated bent SiGe film demonstrating the increased bending curvature [compared to (e)] as the surface stress acts in concert with the misfit strain. (g) The surface dimers and surface stress configurations are switched as compared to (f), now opposing the misfit strain to induce a bending in the opposite direction. Arrows mark stress directions. Reprinted from ref. 36 with permission.

photolithography can be used to pattern many identical structures, suitable for large-scale production. E-beam lithography can be used to pattern individual structures with much smaller dimensions. After patterning, the $2 \mathrm{D}$ strained membrane is released from the substrate and rolled up into 3D structures by etching away the underlying sacrificial layer. As mentioned in Sec. 1, the etching rate of the sacrificial layer is one of the extrinsic parameters that defines the desired ultimate 3D structure.

Real-time video microscopy reveals that the rolling-up process is highly nonlinear at the beginning, linear at the intermediate stage, and stops for long etching time. ${ }^{9,59}$ A nonlinear rolling-up process implies a fast mean etch rate, whereas a linear roll-up process would imply a low mean etch rate. Fig. 11 shows a detailed evolutionary view of the process. ${ }^{9}$ All etching processes start at zero time; the time bar shows the beginning and ending of membrane deformation, respectively, when they are observable.

The etching rate has a strong influence on the shape of tubes. Fast etching leads to an asymmetric rolling of tubes while slower etching leads to more symmetric rolling of tubes. When the membrane size is sufficiently large, the degrees of freedom in rolling increase, as well as the possibility of asymmetric rolling, ${ }^{9}$ as shown in Fig. 11. Moreover, the fast etching process has a linear dependence on the membrane dimension (etching length), which indicates that the mass transport of chemicals is fast enough so that etching is mainly determined by the chemical reaction at the etch front, namely a kinetically controlled etch rate. Conversely, the slow etching process has a nonlinear dependence on the etching length, indicating that the etch rate is transport-controlled. This transport dependence may explain why, in Fig. 11, for the slow processes the $70 \times 70 \mu \mathrm{m}^{2}$ bar is shorter than the $60 \times 60 \mu \mathrm{m}^{2}$ one.

Precise positioning of rolled-up tubes can be achieved by controlling the etch rate as well. ${ }^{59} \mathrm{~A}$ slow etch rate is the general rule to achieve symmetric rolling of membranes and precise positions of the rolled-up structures over a large area.

\section{Fabrication methods}

The fabrication of freestanding rolled-up 3D micro- and nano-objects generally consists of three critical steps: (1) the formation of nanometer-thick single- and multilayer strained membranes with defined and generally maximal strain gradient, (2) patterning of the strained membranes into desired geometries, and (3) releasing the patterned membranes from the underlying substrate by selective etching of a sacrificial layer. More specifically, conventional optical lithography and/or e-beam lithography and other nanopatterning techniques can be used to pattern strained membranes into various geometries as well as along different directions. After lithography, the desired patterns are transferred onto strained membranes by using reactive ion etching (RIE). A rinsing/ drying step can be added after selective etching. Because they are based on standard device fabrication technology, one can expect the processes to create these rolled-up structures to be highly scalable.

\subsection{Establishing and tuning strain}

Strain modifies the Si band structure by splitting the conduction and valence band valleys or maxima. This band splitting suppresses intervalley scattering and reduces the effective transport mass, resulting in significant carrier mobility enhancement and hence has been used to improve the performance of semiconductor devices and to advance significantly the field of Si-based quantum electronics. ${ }^{60}$ In contrast, strain relaxation is the driving force behind the rolling-up technology, i.e., formation of 3D nanostructures from 2D patterned sheets. In general, the strain can be created by the following methods: (1) via the lattice mismatch between different crystalline materials with an epitaxial relationship, (2) via non-epitaxial deposition, (3) via differential thermal expansion of two materials, (4) via differential swelling of materials, or (5) in very thin membranes, via differential surface stress, as mentioned in Sec. 2.2.2. 


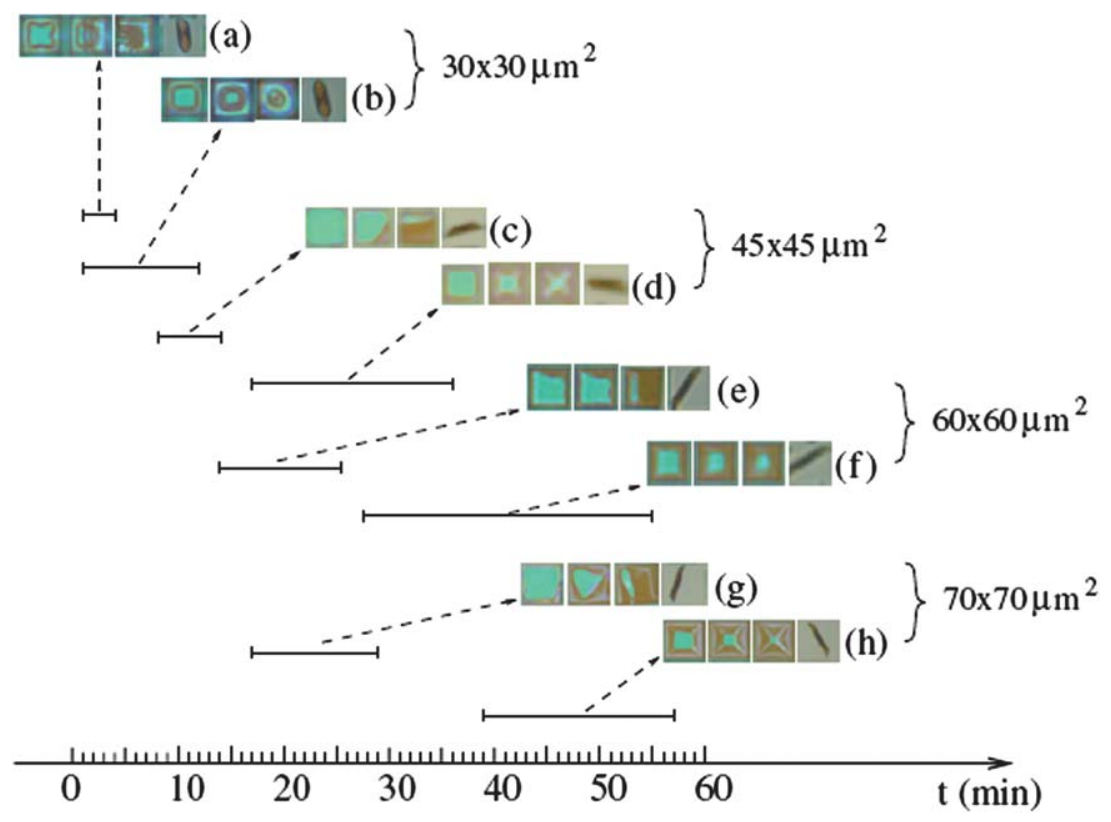

Fig. 11 Snapshots of two different etching processes. Fast tube formation from a $30 \times 30 \mu \mathrm{m}^{2}$ square, a $45 \times 45 \mu \mathrm{m}^{2}$ square, a $60 \times 60 \mu \mathrm{m}^{2}$ square, and a $70 \times 70 \mu \mathrm{m}^{2}$ square are shown in (a), (c), (e), and (g), respectively. Correspondingly, much slower processes are shown in (b), (d), (f), and (h). The time bar shows the beginning and ending of membrane deformation, respectively; when they are observable. The fast process is kinetically controlled and is linearly dependent on the square size. In contrast, the slow process is transport controlled and is nonlinearly dependent on the square size. Reprinted from ref. 9, with permission.

3.1.1 Heteroepitaxial strain. A wide spectrum of heterostructures has been used to make strained films for rolled-up objects, ranging from Group IV materials $(\mathrm{Si} / \mathrm{Ge})^{7-9,11-13,28,48,62-68}$ and III-V materials (GaAs/InAs), ${ }^{15,39-42,68-72}$ to II-VI materials $(\mathrm{CdS} / \mathrm{CdSe}){ }^{73}$ These heterostructures are typically grown by epitaxy. Epitaxy is growth of a single-crystalline film on a singlecrystalline substrate. Homoepitaxy is growth on the substrate of the same material, while heteroepitaxy is growth on the substrate of a different material. In heteroepitaxy, there exists a misfit strain at the interface caused by the lattice mismatch between the two materials. For example, suppose material A has a larger lattice constant than that of material B. When material B is epitaxially grown on material $\mathrm{A}$, the lattice of $\mathrm{B}$ is stretched to fit the lattice of $A$ at the interface. As a result, a tensile strain is created in material B. The opposite (A grown on B) causes a compressive strain in material A.

We focus on the Group IV materials here. The heteroepitaxial growth of $\mathrm{Si} / \mathrm{Ge}$ heterostructures is well established. There is a $4 \%$ lattice mismatch between $\mathrm{Si}$ and Ge. Ideally, one can grow a Ge layer epitaxially onto a $\mathrm{Si}(001)$ substrate to create a $4 \%$ misfit strain at the $\mathrm{Si} / \mathrm{Ge}$ interface if the Ge is fully strained. In practice, the strain is so large that the consequences of strain are already evident with the deposition of fraction of a monolayer of the different material. For example, scanning tunneling microscopy (STM) shows the formation of linear defects (misfit dislocations in the surface plane) when pure Ge is deposited on pure $\mathrm{Si}^{74,75}$ With subsequent growth of pure Ge, coherent 3D nanocrystals form in what is known as the Stranski-Krastanov growth mode $^{76}$ (initial 2D layer growth, also called the wetting layer, followed by $3 \mathrm{D}$ island growth). These 3D nanocrystals can relieve strain because, even though they are coherent with the substrate, their lattice constant can increase vertically, effectively relieving strain. The 3D nanostressors, termed "huts", ${ }^{1}$ have four (105) facets whose structure has been thoroughly investigated. ${ }^{1,77}$ Fig. 12 shows a near-atomic-resolution STM image of one such 3D hut, and also shows a cross-section transmission electron microscopy (XTEM) image of the same structure. ${ }^{33}$

In addition to the formation of $3 \mathrm{D}$ coherent nanostressor islands as above, a strained film growing on a rigid substrate can reduce built-in strain by a competing mechanism, via dislocation formation, the more conventionally known way. The strain energy tends to be released plastically by dislocations when the strain is relatively small, i.e., for a low-Ge-concentration $\mathrm{SiGe}$ alloy on $\mathrm{Si}(001) .{ }^{78}$ For films with larger misfit strain, e.g., Ge on $\mathrm{Si}(001)$, the system prefers $3 \mathrm{D}$ island formation after the 2D-film critical thickness is exceeded, if the temperature is high enough to allow the required diffusion and nucleation kinetics. The nucleation barrier for a $3 \mathrm{D}$ coherently strained island ${ }^{79}$ decreases rapidly with strain as $\varepsilon^{-4}$, while that for dislocations is linear with $\varepsilon^{-1}$. Hence for large strain (high Ge concentration, in this case), $3 \mathrm{D}$ island formation is the preferred path to strain energy reduction.

In the creation of strain-engineered nanoarchitectures, we want in general to maximize the strain gradient and to be able to tune the strain to the desired level, which implies that we generally want to avoid unknown plastic strain relaxation, and we generally want a flat film rather than hut formation. Therefore, a practical way to form strained $\mathrm{Si} / \mathrm{Ge}$ bilayer films is to grow low-Ge-concentration $\mathrm{SiGe}$ alloy on a $\mathrm{Si}(001)$ substrate, for which the preferential growth is as a $2 \mathrm{D}$ layer. The magnitude of misfit strain can be tuned by varying the composition of the SiGe alloy layer, but there are limitations. In order to keep the SiGe alloy layer fully strained, its thickness is always kept below the kinetic critical thickness for the formation of dislocations for that 


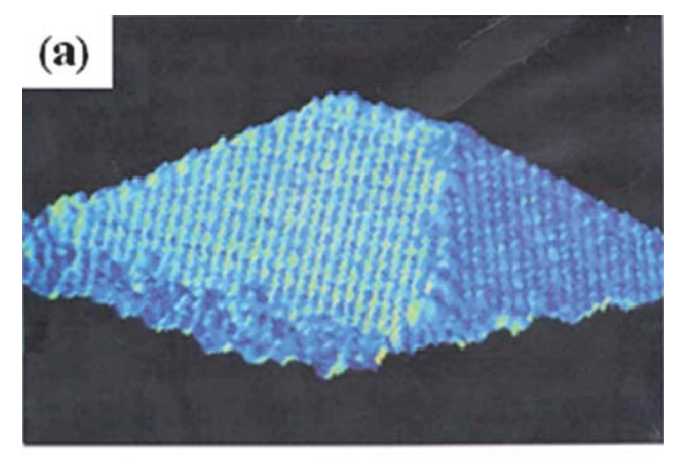

(b)

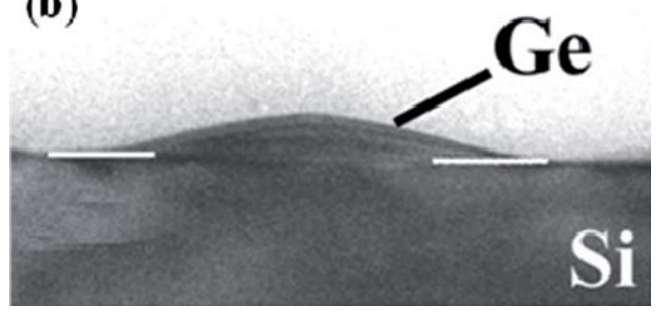

Fig. 12 Ge "hut" nanostressor on Si(001). (a) STM image; (b) XTEM image: the white lines show the Ge hut-Si substrate interface. Reprinted from ref. 33 with permission. Images courtesy of Y.-W. Mo and E. Sutter.

composition. ${ }^{80,81}$ There are added complications in determining the strain gradient and the maximum strain. Essentially all prior effort has focused on epitaxy on substrates that are much thicker than the growing film, and the models for dislocation formation referenced above relate to that situation. It is only quite recently that systems have been considered in which the substrate is not thick. The way the strain is shared between the growing film and substrate begins to change as the substrate gets thinner. The growing strained film applies effectively an "external" stress to bend and stretch the substrate (Fig. 2). To remain coherent, i.e., not plastically relaxed, the growing film in the conventional system must always be below the critical thickness for dislocation formation, i.e., much thinner than the substrate. When the deposited film is much thinner than the substrate, bending dominates (even though it is ignored because it is so small) and increases linearly with increasing film thickness, following Stoney formula ${ }^{44}$ as shown in Fig. 2(a). The behavior when the film thickness becomes comparable to the substrate thickness deviates dramatically from the Stoney formula linear dependence. The behavior now follows the classical Timoshenko formula, as shown in eqn (1) in Sec. 2.1, and can be understood by considering the evolution of strain sharing between the film and substrate (Fig. 2). Strain sharing affects critical thicknesses and defect formation in heteroepitaxy and produces most interesting results in nanoepitaxy.

Using a thin, freestanding substrate allows better strain sharing between growing film and substrate and makes it possible to grow a thicker, fully strained, unrelaxed film on it; whereas the same film grown on a bulk substrate is relaxed via the formation of dislocations. That is so because the thin substrate shares elastically in the strain, by distorting via bending or changing linear dimensions. By doing so, one can maximize the strain gradient and tune the strain to the desired level.
A complication that is not obvious or easily understood works in the opposite direction. If the thin substrate is, in fact, held on a carrier such as the oxide in silicon-on-insulator (SOI) substrate, then it is neither freestanding, nor is the system the same as bulk. The thin substrate in this configuration cannot readily share strain elastically until the oxide is etched and the bilayer structure is released. That has the following consequence: the thermodynamic critical thickness for plastic relaxation actually is lower than for growth of the same layer on a bulk substrate. The reason is that the energy associated with dislocation formation is lower at the oxide/crystalline interface ${ }^{82}$ and thus the critical thickness is lower. This factor affects the degree of strain one can introduce starting with layered structures such as SOI.

Many possibilities exist for the preparation of substrates for strained-layer Si/Ge growth and subsequent growth. We describe an example. The Si substrate is cleaned with diluted hydrofluoric acid (HF) to etch off the native oxide on the surface, followed with a piranha clean $\left(\mathrm{H}_{2} \mathrm{SO}_{4} / \mathrm{H}_{2} \mathrm{O}_{2}\right)$ in $10 \mathrm{~min}$, and a diluted $\mathrm{HF}$ etch for a few seconds to remove the oxide layer formed during the piranha clean. Epitaxial growth can be most readily achieved via molecular beam epitaxy (MBE) or chemical vapor deposition (CVD), which both provide a precise control of film thickness in the monolayer range. For ultrahigh-vacuum MBE or CVD (UHV-MBE/CVD) growth, the sample is cleaned with an additional 15-minute $\mathrm{SC} 1$ clean $\left(\mathrm{NH}_{4} \mathrm{OH} / \mathrm{H}_{2} \mathrm{O}_{2} / \mathrm{H}_{2} \mathrm{O}\right.$-mixture) at $\sim 80^{\circ} \mathrm{C}$ and dipped deliberately into diluted $\mathrm{HF}$ acid to terminate the surface with hydrogen before transfer to the MBE or CVD chamber, in order to prevent the growth of native oxide. The epitaxial $\mathrm{Si} / \mathrm{SiGe}$ alloy layer is then grown, for example, onto a $\mathrm{Si}$ (001) substrate. The growth temperature is generally $\sim 600^{\circ} \mathrm{C}$ or lower, measured by an optical pyrometer. The Ge composition in the SiGe layer can be varied by changing Ge flux rate in MBE or germane flow rate in CVD, i.e., changing the Ge content in the gas phase. The lower growth temperature prevents the nucleation of dislocations via surface roughening, as surface roughening is
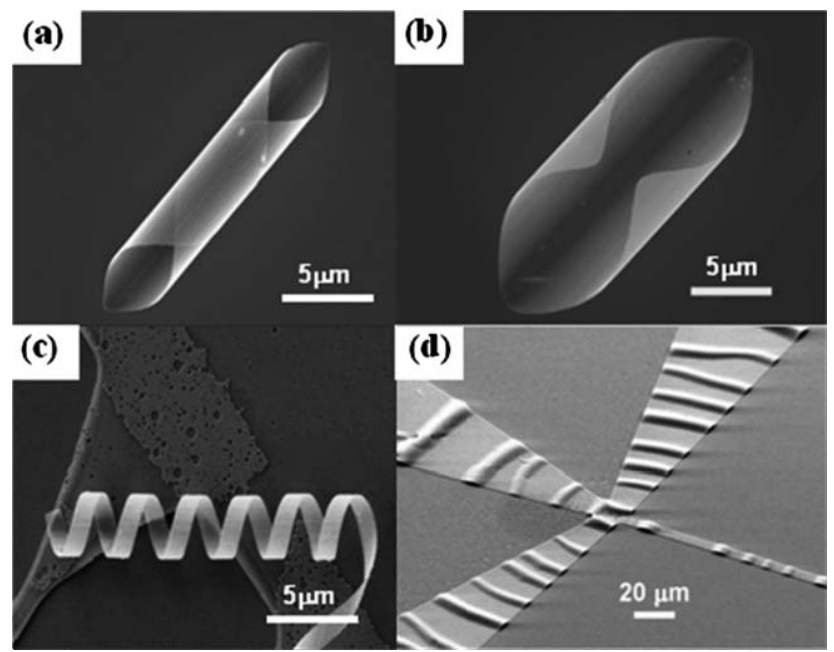

Fig. 13 A variety of rolled-up structures fabricated from all-semiconductor strained layers (bilayer and multilayer). (a)-(c) A closed, a partially opened microtube and a microcoil fabricated from $\mathrm{Si} /$ $\mathrm{Si}_{0.8} \mathrm{Ge}_{0.2}(19 \mathrm{~nm} / 30 \mathrm{~nm})$ (d) Wiggles fabricated from a $\mathrm{Si}_{1} / \mathrm{Si}_{0.8} \mathrm{Ge}_{0.2} / \mathrm{Si}$ $(19 \mathrm{~nm} / 30 \mathrm{~nm} / 20 \mathrm{~nm})$ strained multilayer. Reprinted with permission from ref. 83 and 84. Images courtesy of Minrui Yu. 
thermally activated. ${ }^{78}$ The composition of SiGe alloy is determined ex-situ by X-ray diffraction. The $2 \mathrm{D}$ growth of the strained $\mathrm{SiGe}$ layer, or the Si layer on top of that, is monitored in situ with reflection high-energy electron diffraction in UHV by following the diffraction pattern of the $(2 \times 1)$ reconstruction. Fig. 13 shows 3D nanostructures ${ }^{83,84}$ that form from epitaxially grown $\mathrm{Si} / \mathrm{SiGe}$ bilayer or multilayer membranes after release from the handle substrate.

In Sec. 2 we briefly discussed single-element membranes with a strain gradient that roll up into micro- and nano-objects. We describe the process more fully here. A strain gradient in a $\mathrm{Si}$ membrane is created by growing a Ge layer on $\mathrm{Si}(001)$ via UHV-MBE at $300{ }^{\circ} \mathrm{C}^{58}$ The low growth temperature ensures the growth of a smooth, fully plastically relaxed Ge layer. A very thin $\mathrm{Si}$ layer is then grown over the Ge. The first few $\mathrm{Si}$ monolayers grown on the Ge layer are highly tensilely strained and then, with increasing $\mathrm{Si}$ thickness, dislocations start to form in the Si to relax misfit strain. Subsequently deposited Si relaxes further, leading eventually to a fully plastically relaxed $\mathrm{Si}$ near the top without relaxing completely the Si deposited first. The result is a tensile-strain gradient in the Si layer with a maximum value at the $\mathrm{Si} / \mathrm{Ge}$ interface and a minimum value at the outer Si surface (partially/fully relaxed, depending on its thickness). The strain graded Si membrane rolls into a microtube after release from the handle substrate by selectively etching the Ge layer. In this method, the fully plastically relaxed Ge layer is both the stressor layer and the sacrificial layer, providing a unique way to form microtubes. The straingraded Si contains dislocations.

The Ge condensation technique ${ }^{67,85,86}$ provides another approach to introduce strain into a membrane system. For example, a 40nm-thick layer of SiGe alloy with uniform $\mathrm{Ge}$ composition of $8 \%$ is grown on silicon-on-insulator (SOI) with a $27 \mathrm{~nm}$-thick template layer and capped with a thin Si layer. ${ }^{67}$ This $\mathrm{Si} / \mathrm{SiGe} / \mathrm{SOI}$ structure is oxidized at $1050^{\circ} \mathrm{C}$ in an oxygen-

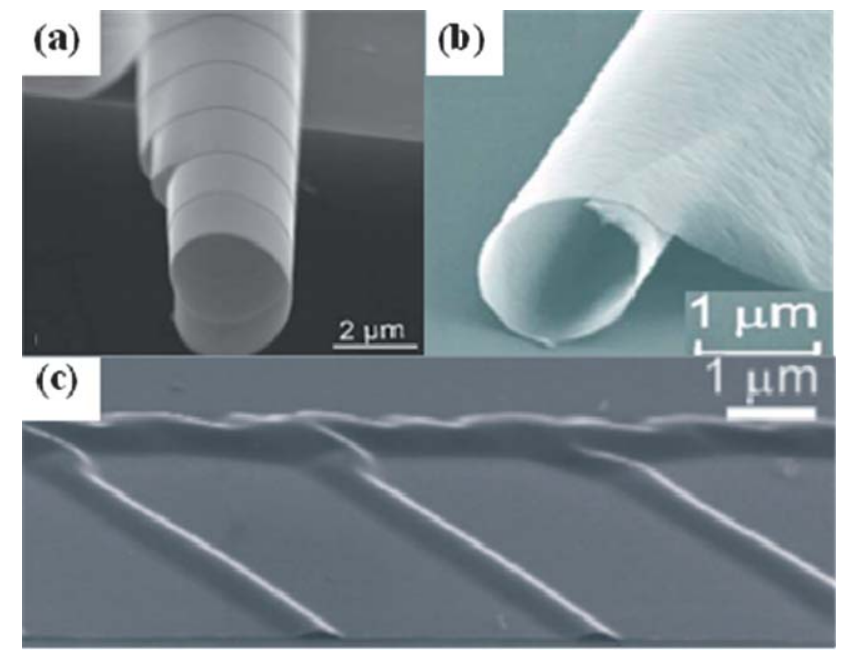

Fig. 14 A variety of rolled-up structures fabricated from single-layer strained semiconductor membranes. (a) A 20nm-thick Si tube with diameter of $2 \mu \mathrm{m}$, (b) A 10nm- thick SiGe tube with diameter of $1.6 \mu \mathrm{m}$. (c) SiGe periodic wrinkles fabricated from SGOI by the Ge condensation technique. Image (a) from ref. 64. Image (b) from ref. 67. Image (c) from ref. 28 with permission. rich environment. $\mathrm{Si}$ in the SiGe alloy is oxidized and $\mathrm{Ge}$ is rejected from the alloy and diffuses into the Si template layer of the SOI substrate. The outdiffusion of Ge during the oxidation is prevented by the top $\mathrm{SiO}_{2}$ layer. As a result, the $\mathrm{Si}$ template layer becomes $\mathrm{SiGe}$ and a gradient of Ge concentration exists in this layer, leading to a compressive-strain gradient in this final $\mathrm{SiGe}$ layer on oxide (called SGOI). Both the Ge distribution profile and the Ge content in the SGOI can be tuned by changing the oxidation time. If all $\mathrm{Si}$ in SGOI is oxidized, GeOI can be made by using this technique. The membrane will, of course, have dislocations in it. If this SiGe layer is released from the oxide, it will curl because of the strain gradient, just as the Si described in the prior paragraph. It can form into microtubes and wrinkles, depending upon the Ge distribution profile, and the patterning and the length of undercut. ${ }^{28,67}$ Fig. 14 shows microtubes and periodic wrinkles formed by rolling up single-layer strained $\mathrm{Si}$ and SiGe films, respectively.

3.1.2 Strain created by non-epitaxial growth. Most thin films of metals, semiconductors, and insulators, deposited by thermal or electron-beam evaporation, ion plating, sputtering, or plasmaenhanced CVD, are non-epitaxial, and will be either polycrystalline or amorphous. Nevertheless they almost always contain built-in stress, making them suitable for stressing other materials. For example, silicon nitride $\left(\mathrm{Si}_{3} \mathrm{~N}_{4}\right)$ is commonly used in the semiconductor device industry as a stressor, both compressive and tensile, depending on the deposition conditions. $\mathrm{Si}_{3} \mathrm{~N}_{4}$ can be deposited by either low-pressure CVD at $\sim 70{ }^{\circ} \mathrm{C}$ from a dichlorosilane/ammonia gas mixture or plasma-enhanced CVD (PECVD) at a lower temperature $\left(250-350{ }^{\circ} \mathrm{C}\right)$ from a silane/nitrous oxide gas mixture. PECVD-deposited nitride can be either tensilely or compressively strained. The film characteristics, as well as the magnitude of intrinsic stress, are determined by the deposition parameters. If a strained $\mathrm{Si}_{3} \mathrm{~N}_{4}$ layer is deposited on a thin substrate to form a bilayer or multilayer

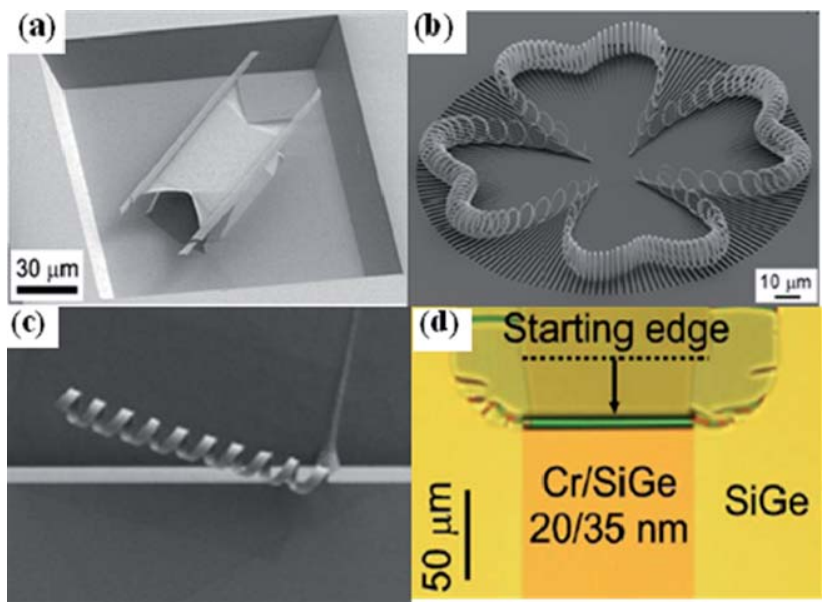

Fig. 15 3D structures fabricated from hybrid strained layers. (a) Two suspended $\mathrm{Si}_{0.6} \mathrm{Ge}_{0.4} / \mathrm{Si} / \mathrm{Si}_{3} \mathrm{~N}_{4} / \mathrm{Cr}(10 / 10 / 10 / 18 \mathrm{~nm})$ tubes with the diameter of $3.8 \mu \mathrm{m}$. (b) SEM image of ringlike structures formed from a $\mathrm{Si} / \mathrm{Cr}$ bilayer. (c) A coil formed from a $\mathrm{Si}_{0.6} \mathrm{Ge}_{0.4} / \mathrm{Si} / \mathrm{Cr}$ trilayer $(11 / 8 / 21 \mathrm{~nm})$. (d) A tube formed from a $\mathrm{Cr} / \mathrm{SiGe}$ bilayer (10/35nm). Image (a) reprinted from ref. 56, images (b) and (c) from ref. 48, and image (d) from ref. 68 with permission. 
membrane, the membrane can, in principle, roll into a tube upon release, if the nitride stress exerted on the other layers is large enough. ${ }^{56}$ Fig. 15(a) shows a pair of microtubes formed from a multimaterial membrane $\left(\mathrm{SiGe} / \mathrm{Si} / \mathrm{Si}_{3} \mathrm{~N}_{4} / \mathrm{Cr}\right)$.

Metal films have also been incorporated with semiconductor layers to form strained multimaterial nanomembrane structures. ${ }^{12,48,56,68}$ The metal layer, for instance chromium $(\mathrm{Cr})$, is deposited on top of semiconductor layers by using thermal or ebeam evaporation. The amount of strain created can be controlled by the evaporation rate. A strain gradient can be achieved in metal layers by low-angle glancing deposition. The diameter of micro- and nanotubes or belts formed from hybrid structures can be modeled by the design rules as described in Sec. 2. Fig. 15(c)-(d) show 3D architectures fabricated from hybrid strained layers.

3.1.3 Thermal strain. Thermal strain arises from the difference in coefficient of thermal expansion (CTE) upon heating or cooling. For example, the functional part in a household thermometer, the bimetallic strip, consists of two different metal layers, such as copper $(\mathrm{Cu})$ and nickel $(\mathrm{Ni})$, laminated together. The temperature is determined from the mechanical bending induced by the differential thermal expansion or contraction of a bimetallic layer when it is heated or cooled.

The same principle can be applied to make wavy Si ribbons on flexible polymer substrates ${ }^{87}$ [e.g., poly(dimethylsiloxane) (PDMS)], as shown in Fig. 16. The CTE of PDMS is approximately two orders of magnitude larger than that of Si. Si membranes can bond to PDMS very well (no delamination even while strained), so the large CTE mismatch between $\mathrm{Si}$ and PDMS can cause a large thermally induced strain when the bilayer is heated or cooled. The large strain can shape Si into different forms. This approach has been demonstrated, as shown in Fig. 16(a). First, the Si ribbons are patterned on SOI by photolithography and RIE, and then released in place by underetching the oxide. Second, a PDMS substrate with thickness of $1-3 \mathrm{~mm}$ is elastically stretched by heating it to temperatures of between $30{ }^{\circ} \mathrm{C}$ and $180{ }^{\circ} \mathrm{C}$. The prestrained PDMS substrate (while it is hot) is bonded with the $\mathrm{Si}$ ribbons. The prestrained PDMS is then released by cooling, leading the PDMS to relax back to its unstrained state. This relaxation leads to the spontaneous formation of wellcontrolled, highly periodic, stretchable wavy structures in $\mathrm{Si}$ ribbons. Fig. 16(b) shows wavy Si ribbons with excellent uniformity over a large area and in a highly reproducible fashion.

3.1.4 Swelling-induced strain. Pure polymer or polymer/metal hybrid materials are more bio-compatible than semiconductors are. Self-rolled polymer and polymer/metal composite microand nanotubes therefore may have potential application in biotechnology. Similar to thermal strain arising from the difference in coefficient of thermal expansion (CTE) upon heating or cooling, swelling-induced strain results from the different swelling properties of chemically dissimilar polymers in selective solvents. The self-rolling process is driven by the selective swelling of the bottom component of the polymeric bilayer. ${ }^{25,26}$ For example, polystyrene and poly (4-vinylpyridine) are a good combination for the polymeric bilayer. ${ }^{26}$ Adding a metal layer on the polymeric bilayer can not only change the diameter of rolledup tube but also alter the tube's electrical and chemical properties. $^{25}$ (a)

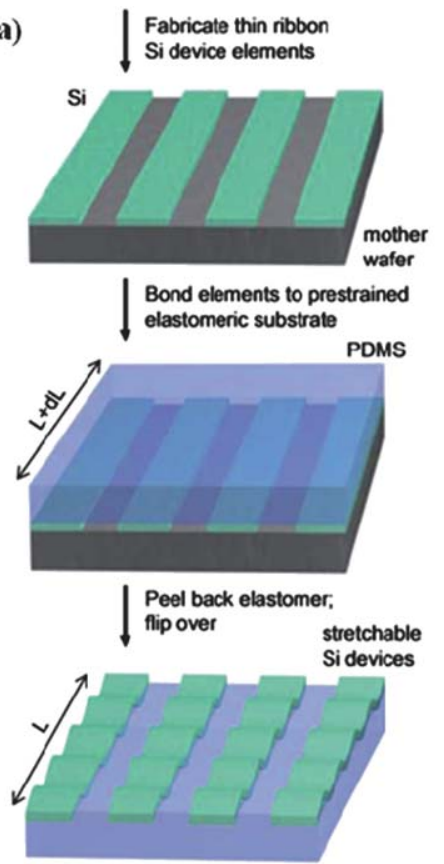

(b)

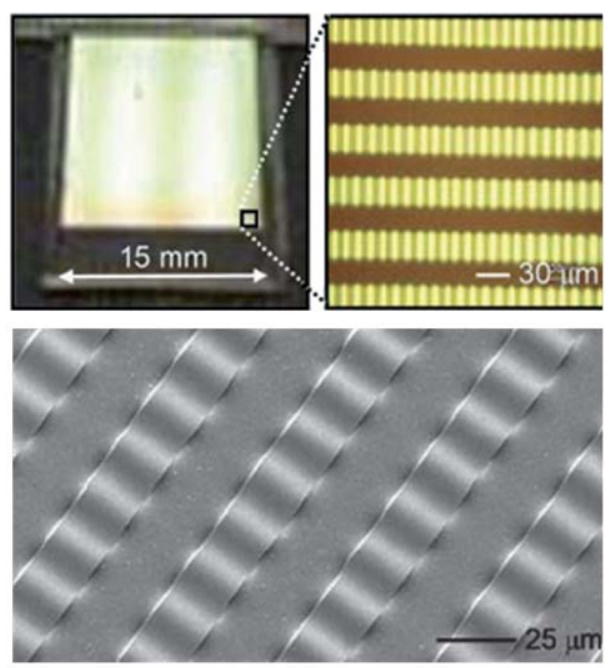

Fig. 16 Arrays of wavy Si ribbons on rubber substrates. (a) Schematic illustration of the process for creating wavy Si ribbons on elastomeric substrates (from top to bottom). (b) Top: Optical images of a large-scale aligned array of wavy Si ribbons (widths $=20 \mu \mathrm{m}, \mathrm{spacing}=20 \mu \mathrm{m}$, thicknesses $=100 \mathrm{~nm}$ ) on PDMS. Bottom: SEM image of four wavy Si ribbons from the array shown on top. Images from ref. 87 with permission. Images courtesy of John Rogers. 
3.1.5 Strain induced by differential surface stress. As mentioned in Sec. 2.2.2, surface reconstruction causes an intrinsic surface stress. For example, the clean $\mathrm{Si}(001)$ surface exhibits a $(2 \times 1)$-type reconstruction, as shown in Fig. 10(a). In an odd-layer membrane, there exists a surface-stress imbalance between its top and bottom surfaces [Fig. 10(d)], creating a unique self-driving force to bend the membrane. Here we discuss the feasibility of observing this proposed bending mechanism experimentally. First, we need an ultrathin freestanding membrane with reconstructed surfaces on the top and bottom of membrane. Ultrathin InAs/GaAs bilayer membranes with few atomic layers have been made, as shown in Fig. 9. Ultrathin Si membranes can be achieved with SOI(001) and thinned to $5 \mathrm{~nm}$ (still too thick) by subsequent thermal oxidation and etching away the thermal oxide. The ultrathin membrane can be patterned and the buried oxide removed by etching to make the membrane freestanding. One would then need to load the sample into a UHV chamber, and obtain $(2 \times 1)$ reconstructed surfaces after removing the chemical and physical adsorbates. Second, a very large region of step-free surface is required. Stepfree regions of $20 \times 20 \mu^{2}$ has been made on $\mathrm{Si}(001)$ by patterning $\mathrm{Si}$ with grating structures and then annealing above $1000{ }^{\circ} \mathrm{C}$ in the UHV chamber. ${ }^{\mathbf{8 8 , 8 9}}$ But doing so on both sides of a free standing membrane seems quite difficult, or impossible, and no experimental demonstration of bending induced by imbalanced surface stresses is likely in the near future.

\subsection{Patterning 2D sheets to make rolled-up structures}

2D strained layers can be patterned into a variety of geometries as well as along different directions. Many identical structures can be made over a large area, depending on the lithographic techniques. Three general methods are reviewed here. The straightforward method to make patterns is mechanical scratching. Scratching can be simply done using a micrometer sized diamond tip. This method provides a quick check of the rolling behavior of a grown layer system in a short processing time. However, the low controllability of this method makes it difficult to have uniform patterns and smooth edges.

Well-controlled patterning is achieved by using either optical or e-beam lithography followed by RIE. The general procedure is as follows: optical/e-beam lithography is used to transfer defined patterns on a photo/e-beam resist film covering the sample surface. Photolithography requires a photomask consisting of a chrome film deposited on a quartz glass. The desired patterns are predefined on it by etching the chrome film. The photomask is inserted between the light source and a resist-covered sample. The patterns are then created on photoresist by exposure and development. The big advantage of photolithography is its high throughput. Many identical patterns can be created over a large area (wafer-level) simultaneously. Light diffraction limits the feature size that can be made by photolithography to opticalwavelength dimensions.

E-beam lithography is used to make features at the nanometer scale by scanning an electron beam across the surface covered by the resist. A predefined mask is not required. E-beam lithography offers flexibility in pattern design and is good for initial characterization of geometric effects on self-rolling. The pattern created in the resist by lithography is then realized in the strained film by
Table 1 Summary of major release approaches for Group IV nanomembranes

\begin{tabular}{|c|c|c|c|}
\hline Functional layer & $\begin{array}{l}\text { Sacrificial } \\
\text { layer }\end{array}$ & Etchant & $\begin{array}{l}\text { Etching } \\
\text { Temperature }\left({ }^{\circ} \mathrm{C}\right)\end{array}$ \\
\hline $\begin{array}{l}\text { Highly doped } \\
\mathrm{Si}(001) \text {-based } \\
\text { materials }\end{array}$ & $\begin{array}{l}\text { Low-doped } \\
\text { Si(001) }\end{array}$ & $\mathrm{NH}_{4} \mathrm{OH}(3.7 \%)$ & 75 \\
\hline $\begin{array}{l}\mathrm{Si}(001) \text {-based } \\
\text { materials }\end{array}$ & Pure Ge(001) & $\mathrm{H}_{2} \mathrm{O}_{2}(30 \%)$ & $25 / 95$ \\
\hline $\begin{array}{l}\mathrm{Si}(001) \text {-based } \\
\text { materials }\end{array}$ & $\mathrm{SiO}_{2}$ & $\mathrm{HF}$ & 25 \\
\hline $\begin{array}{l}\text { Most inorganic } \\
\text { materials }\end{array}$ & $\begin{array}{l}\text { Polymer } \\
\quad \text { (photoresist) }\end{array}$ & acetone & 25 \\
\hline
\end{tabular}

using dry etching (RIE). In this process a reactive chemical gas interacts with the surface by using a combination of chemical and physical etching processes. Etch gas atoms are accelerated toward the substrate by a large radio frequency voltage applied in the system and, upon contact, chemically react with the surface while also physically removing material due to their high kinetic energy. RIE is known for its excellent vertical sidewall etching (anisotropic) properties, avoiding the undercut issue in wet etching.

\subsection{Selective etching}

The fabrication of freestanding rolled-up structures requires releasing the strained membranes from the underlying substrate by selective etching. A sacrificial layer is introduced to facilitate this release. The key factor is the etching selectivity between a sacrificial layer and the membrane. In principle, the sacrificial layer must etch away without etching/damaging the membranes. A high etching selectivity is crucial for a successful release, putting constraints on materials selection.

Several release approaches to achieve high etching selectivity to fabricate Group IV semiconductor nanomembranes have been demonstrated. $7-9,11-13,28,48,62-68$ Four major approaches are summarized in Table 1 and briefly described here.

- Approach 1: Tubes and coils can be formed from a strained highly boron doped $\mathrm{Si} / \mathrm{SiGe}$ bilayer with low-doped Si serving as the sacrificial layer. ${ }^{7,12,13}$ An ammonium hydroxide-water solution $(3.7 \%)$ is used as the selective etchant because it can etch away low-doped Si at $75^{\circ} \mathrm{C},{ }^{7} 8000$ times faster than highly doped $\mathrm{Si}$, with boron concentration as high as $10^{20} \mathrm{~cm}^{-3}$. The etch stop effect is more distinct if a SiGe film is used, because the etching selectivity between $\mathrm{Si}_{0.9} \mathrm{Ge}_{0.1}$ and $\mathrm{Si}$ is better than $80 .{ }^{62}$

- Approach 2: Tubes can be rolled up from a strained $\mathrm{Si} / \mathrm{SiGe}$ layer grown onto a Ge buffer layer, serving as a sacrificial layer. ${ }^{8,58,63}$ The Ge buffer layer is selectively etched away with $30 \% \mathrm{H}_{2} \mathrm{O}_{2}$ at room temperature ${ }^{58}$ or at $90{ }^{\circ} \mathrm{C}$. ${ }^{8}$ The temperature will determine the differential etch rate, which is critical in the release.

- Approach 3: $\mathrm{SiO}_{2}$ is the most commonly used sacrificial layer for Si membranes. The advantage of using SOI is the very high selectivity of etching between $\mathrm{Si}$ and $\mathrm{SiO}_{2}$, in contrast to the use of heavily boron-doped $\mathrm{Si}$ or $\mathrm{Ge}$ as sacrificial layers. A compressively strained $\mathrm{SiGe}$ alloy grows epitaxially on SOI below the kinetic critical thickness for formation of dislocations. The SiGe/Si bilayer would bend or roll naturally downward upon 
release. This is because compressively strained SiGe would like to expand while tensilely strained $\mathrm{Si}$ would like to shrink, resulting in a net downward bending moment, as shown in Fig. 2. The spacing for bending is limited by the thickness of the buried oxide, putting a constraint on the bending process. In order for the $\mathrm{SiGe} / \mathrm{Si}$ strained bilayer to bend or roll upward upon release from the SOI substrate, an extra thin Si layer is grown on top of the $\mathrm{SiGe} / \mathrm{Si}$ bilayer to form a $\mathrm{Si} / \mathrm{SiGe} / \mathrm{Si}$ trilayer membrane. The top Si layer has to be thinner than the bottom Si layer to ensure upward bending. The bending curvature decreases (the diameter of tube increases) when the total film thickness increases as predicted by eqn (1) in Sec. 2.1. Alternatively, if one started with SGOI, described in Sec. 3.1.1, tensilely strained $\mathrm{Si}$ can be grown on it. The $\mathrm{Si} / \mathrm{SiGe}$ strained bilayer will bend or roll upward upon release, because an upward bending moment is created (Si grown on SGOI). The advantages of using SGOI over SOI as a substrate is the bending is upward without space limitation and tubes with smaller diameters are possible because the $\mathrm{Si} / \mathrm{SiGe}$ bilayer is thinner than $\mathrm{Si} / \mathrm{SiGe} / \mathrm{Si}$ trilayer. The drawback is that there are dislocations in SGOI.

- Approach 4: The requirement of high etch selectivity between a sacrificial layer and strained membranes restricts the material choices. Recently, a new approach, using a polymer layer as a sacrificial or template layer, has been developed to overcome this limitation. ${ }^{25,26}$ As mentioned earlier, most deposited thin films contain stress and a stress (strain) gradient. If such a film (it will be non-single crystal) is deposited onto a polymer sacrificial layer, such as a photoresist, it will contain either compressive or tensile strain. These films will be likely elastically isotropic materials. The strain gradient can be created in single layers or multilayers. In either case, the strained layer, released from the substrate by removing the photoresist (e.g., with acetone), will form micro- or nanotubes or wrinkles, depending on the magnitude of the strain gradient and the geometric conditions (Sec. 2.1).

The uniqueness of this approach is that acetone can remove a polymer layer with $100 \%$ selectivity over almost all inorganic materials. The approach makes it possible to roll up a wide spectrum of materials and materials combinations. Moreover, the diameter of microtubes can be tuned by varying the thickness and built-in strain of deposited films. This approach has limitations. Only polycrystalline or amorphous inorganic membranes can be deposited on a polymer layer and they must be deposited at very low substrate temperature to prevent the decomposition of the polymer.

Fig. 17 shows microtubes fabricated via these four major approaches. For all of them, it is clear that the differential etching rate is important. Less clear is that the absolute etching rate is equally important, as the final rolled-up structure is determined by the rate of strain relaxation relative to the absolute etching rate. Several experiments have demonstrated that the rolling process is strongly dependent on the etching rate, $, 95,72$ as described in Sec. 2.3.

\subsection{Large-scale fabrication}

Large-scale fabrication of identical strained-membrane nanomechanical architectures not only depends on the intrinsic geometric and physical conditions as described in Sec. 2, but also

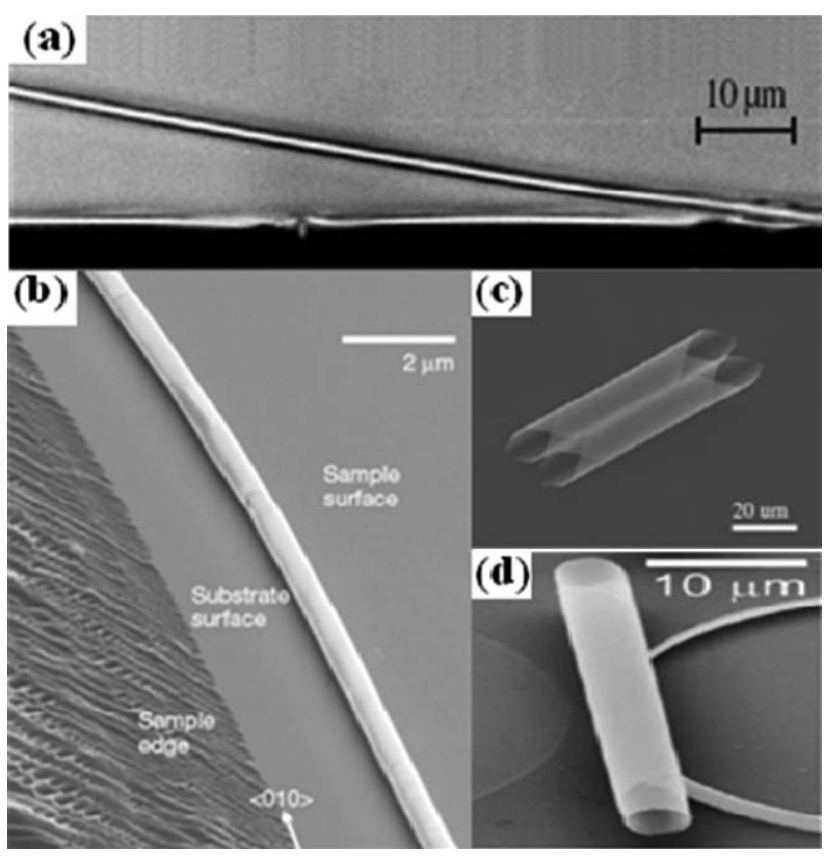

Fig. 17 SEM images of tubes formed via different releasing approaches. (a) A highly B-doped $\mathrm{Si}_{0.6} \mathrm{Ge}_{0.4} / \mathrm{Si}(20 / 10 \mathrm{~nm})$ tube released via approach 1 described in the text. (b) An undoped $\mathrm{Si}_{0.8} \mathrm{Ge}_{0.2} / \mathrm{Si}(6 / 1 \mathrm{~nm})$ tube released via approach 2. (c) Two $\mathrm{Si}_{0.8} \mathrm{Ge}_{0.2} / \mathrm{Si}(50 / 20 \mathrm{~nm})$ tubes released from SGOI via approach 3. (d) $\mathrm{A} \mathrm{SiO}_{2} / \mathrm{SiO}$ tube released from a polymer sacrificial layer via approach 4. Image (a) from ref. 61. Image (b) from ref. 8. Image (c) from ref. 9. Image (d) from ref. 26 with permission.

relies on extrinsic processing parameters as described in Secs. 3.1 and 3.2. In order to fabricate many identical nanoarchitectures simultaneously, a controllable and reliable fabrication process is required., ${ }^{9,72}$ The nanomechanical-architecture approach is compatible with standard device fabrication technology. Strained nanomembranes are created via CVD growth with a precise control of film uniformity. Conventional lithography is performed to pattern the strained membrane into many identical patterns, which are then realized by RIE. Release is performed with selecting etching. Etching uniformity is necessary to obtain consistent tube or other structure formation over a large area. Uniform etching over a large area can be achieved in a controlled wet-etching process. Fig. 18 shows arrays of $\mathrm{Si} / \mathrm{SiGe}$ microtubes that have been made in parallel by following the procedure described here. The large-area fabrication of InAs/GaAs nanotubes has also been demonstrated. ${ }^{72}$ Uniform-etching processes are described in ref. 9 and 72 .

\section{Properties of membrane-based 3D nanoarchitectures}

The nanomechanical architectures described in Secs. 2 and 3 combine the excellent properties of their constitutive materials with their unique 3D geometry, with significant implications for their use as electro- and optomechanical sensors, and in electronic, optoelectronic, and nanofluidic applications. In this scenario a detailed knowledge of structural, mechanical, electronic, optical, and surface properties of rolled-up films is a prerequisite. 
(a)

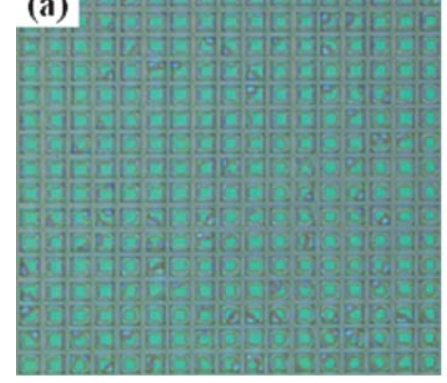

(b)

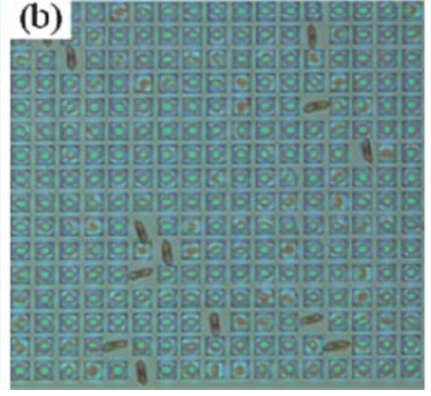

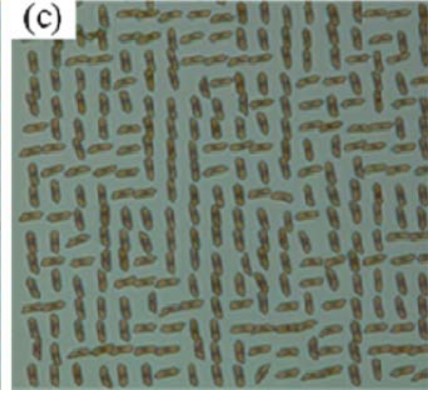

Fig. 18 An array of 270 tubes rolled up from $\mathrm{SiGe} / \mathrm{Si}$ bilayer squares $(30 \mu \mathrm{m} \times 30 \mu \mathrm{m})$ by underetching of $\mathrm{SiO}_{2}$ in $\mathrm{HF}$ solution for $13.2 \mathrm{~min}$. Snapshots at $20 \mathrm{~s}, 10 \mathrm{~min}$, and $13.2 \mathrm{~min}$ are shown in (a), (b), and (c), respectively. Reprinted from ref. 9 with permission.

A significant effort has indeed been made in the characterization of various kinds of rolled-up structures. Detailed structural characterization of tube walls, performed by scanning and transmission electron microscopy (SEM/TEM) reveals that semiconductor membranes rolled into tubes with diameters in the micro- and sub-micrometer range preserve their single-crystal nature, and that defects form in a tube only where the rolling of the membrane involves misorientation. ${ }^{39,42,59,61}$ Good structural stability of various rolled-up objects (i.e., tubes, rings, and spirals) subjected to mechanical ${ }^{12,13,90}$ and thermal stresses ${ }^{17,42}$ has been reported. Electrical conductivity in a bent membrane has been measured and proven to be in the same range as for a flat film. ${ }^{17}$ The magnetotransport in electron systems formed in curved membranes has been investigated. ${ }^{15,65,91}$ Visible photoluminescence (PL) has been observed at room temperature from $\mathrm{Si}$ nanostructures embedded in a cylindrical solid matrix. ${ }^{16,20,92}$ The intensity of the PL signal acquired from rolled-up films, including $\mathrm{Si}$ nanostructures, is of the same order of magnitude. Rolled-up tubes can act as optical ring resonators, because of their circular cross-section. ${ }^{16}$ More recently, optical wave guiding in $\mathrm{Si} / \mathrm{SiO}_{\mathrm{x}}$ tubes has also been demonstrated. ${ }^{92}$ A novel radiation emission mechanism has been proposed in wrinkled $\mathrm{SiGe} / \mathrm{SiGe}$ membranes. ${ }^{93}$ A local-wetting induced deformation of $\mathrm{SiGe} / \mathrm{Si}$ curled membranes has been demonstrated. This deformation suggests that distinctive far-field radiation patterns in the terahertz (THz) range could be achieved. ${ }^{84}$ In this section, we briefly review the results that lead to the conclusions listed above.

\subsection{Structural properties}

A detailed study of the wall structure of rolled-up tubes has been reported for all-semiconductor and hybrid structures. Scanning and transmission electron microscopy (SEM/TEM), electron energy loss and secondary-ion mass spectroscopy (EELS and SIMS), as well as micro-Raman spectroscopy, have been performed for this purpose. This section focuses on characterization of all-semiconductor group-IV structures.

In early work, ${ }^{61}$ high-resolution TEM (HRTEM) was performed to demonstrate the single-crystalline structure of a $\mathrm{Si}_{0.2} \mathrm{Ge}_{0.8} / \mathrm{Si}_{\mathrm{Si}} \mathrm{Si}_{0.2} \mathrm{Ge}_{0.8}$ trilayer rolled-up tube with an inner diameter of $10 \mathrm{~nm}$. Fig. 19 shows TEM images of a portion of the tube freestanding over the edge of the substrate. A HRTEM image of the tube wall [Fig. 19(b)] clearly shows the single-crystal structure of the bent trilayer with $\{111\}$ atomic planes spaced by 3.1 A. Recent advances in TEM sample preparation have
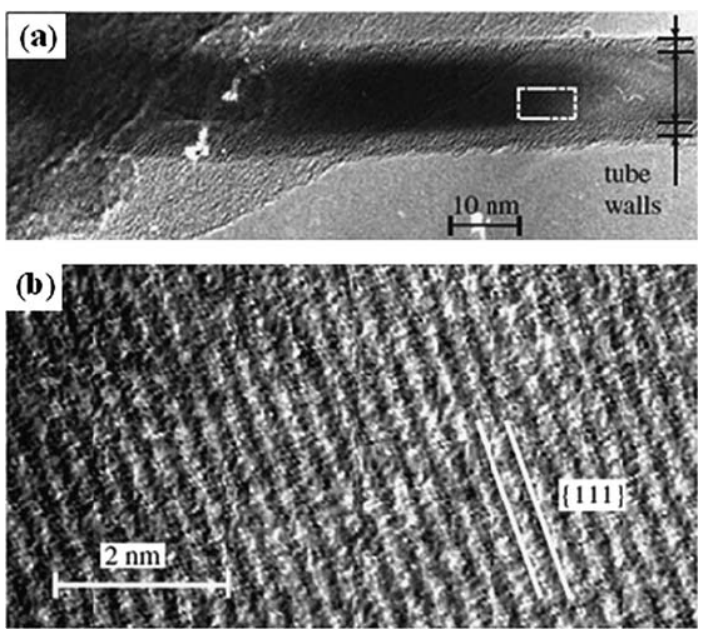

Fig. 19 TEM images of a freestanding rolled-up tube. (a) Plane-view HRTEM image of a $1 \mathrm{~nm} \mathrm{Si}_{0.2} \mathrm{Ge}_{0.8} / 1 \mathrm{~nm} \mathrm{Si} / 0.5 \mathrm{~nm} \mathrm{Si} \mathrm{Si}_{0.2} \mathrm{Ge}_{0.8}$ tube with a diameter of $10 \mathrm{~nm}$. (b) Zoomed-in image of the area denoted by the rectangle in (a). Reprinted from ref. 61 with permission.

allowed cross-sectional TEM (XTEM) of rolled-up tubes and investigation of the elemental distribution across the tube wall by spatially resolved EELS. ${ }^{16,39,59}$ This technique has been employed to demonstrate that the walls of the rolled-up tubes formed by multiple rotations of the semiconductor membranes alternate crystalline semiconductor and thin amorphous-oxide layers. A detailed description of the fabrication process and of the characterization of this structure is reported in ref. 16 .

The prospect of integrating Group IV based tubes with mainstream Si electronics to make lab-on-chip devices requires considering the structural stability of rolled-up layers at high annealing temperatures. The thermal stability of $\mathrm{B}$ doped $\mathrm{Si}_{0.67} \mathrm{Ge}_{0.33} / \mathrm{Si}$ tubes was investigated via local laser heating combined with SEM and Raman spectroscopy. ${ }^{42}$ Approach 1 described in Sec. 3.3 for releasing the bilayer was used. The TEM image and selected-area electron diffraction pattern reveal the good crystal quality of a single freestanding tube. ${ }^{39}$ SIMS profiles indicate that uniform $\mathrm{B}$ doping with a concentration of $\sim 1 \times 10^{20}$ $\mathrm{cm}^{-3}$ exists in a $9 \mathrm{~nm} / \mathrm{Si}_{0.67} \mathrm{Ge}_{0.33} / 17 \mathrm{~nm} \mathrm{Si}$ bilayer. The wall thickness measured by SEM $(17 \mathrm{~nm})$ was thinner than the value derived from SIMS data ( 26nm), as shown in Fig. 1 in ref. 42. This discrepancy was attributed to an insufficient etching selectivity. As a result, the bilayer was partially etched during the 
release processing, which led to a lower wall thickness, demonstrating that high etching selectivity is crucial for a controlled release with predictable outcomes.

Fig. 20 shows the $\mathrm{Si}_{0.67} \mathrm{Ge}_{0.33} / \mathrm{Si}$ tube collapses after local laser annealing at an excitation power of $6.7 \mathrm{~mW}$ for $5 \mathrm{~min}$. Raman spectroscopy is used to measure the vibrations of chemical bonds in materials. Heating a material causes a change in vibration frequency and a Raman peak shift and so Raman spectroscopy can be used to characterize the thermal stability of tubes. ${ }^{42}$ Fig. 21 shows Raman spectra as a function of excitation power and corresponding tube wall temperature. The laser annealing is performed in air. At low excitation power $(\leq 0.7 \mathrm{~mW})$, the measured Raman spectra are dominated clearly by separated peaks related to the vibration modes of the $\mathrm{Si}-\mathrm{Si}$ bond in the $\mathrm{Si}$ and SiGe layers of the tube wall. There is no observable shift of the $\mathrm{Si}-\mathrm{Si}$ peak at the low excitation power, indicating that no significant heating of the tube occurs. A tensile-strain gradient in the Si layer of the tube wall causes the Si-Si peak of the Si layer to broaden and shift to $517 \mathrm{~cm}^{-1}$, relative to the peak of Si-Si bonds in the unstrained substrate at $520 \mathrm{~cm}^{-1}$.

When the excitation power is increased to $1.9 \mathrm{~mW}$ or above, heating causes the $\mathrm{Si}-\mathrm{Si}$ peaks from the $\mathrm{Si}$ and $\mathrm{SiGe}$ layers to shift toward lower wave numbers. These two $\mathrm{Si}-\mathrm{Si}$ peaks merge at high excitation power. The Si-Ge and Ge-Ge peaks in the SiGe layer become more pronounced after heating, indicating the tube structure is changed because of interdiffusion of $\mathrm{Si}$ and $\mathrm{Ge}$, and due to Ge condensation in the annealed area. The tube wall structure starts to become unstable. Fig. 21(b) shows the temperature corresponding to an excitation power of $6.7 \mathrm{~mW}$ is $925^{\circ} \mathrm{C}$, i.e., a temperature high enough to promote a significant $\mathrm{Si}-\mathrm{Ge}$ interdiffusion and Ge condensation in the tube wall. The tube is then broken after heating at a high temperature for several minutes, as shown in Fig. 20. The trend of degradation of the tube structure, according to the shifts of $\mathrm{Si}-\mathrm{Si}$ peaks caused by thermal heating, is summarized in Fig. 21(b). The detailed Raman spectroscopy is described in ref. 42 .

\subsection{Mechanical properties}

The excellent mechanical strength of a $\mathrm{Si} / \mathrm{SiGe}$ microspiral was demonstrated qualitatively in early work. ${ }^{61}$ It can be bent through an angle $>90^{\circ}$ and can be significantly stretched along its axial direction without fracture. ${ }^{61}$ This behaviour indicates the microspiral is extremely elastic. A quantitative study of
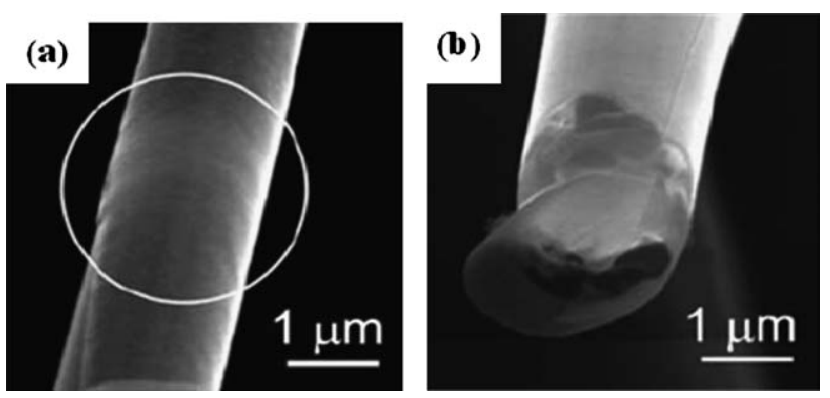

Fig. 20 SEM images of single freestanding tube rolled from a $9 \mathrm{~nm}^{-} \mathrm{Si}_{0.67} \mathrm{Ge}_{0.33} / 17-\mathrm{nm}$ Si bilayer, before (a) and after (b) annealing at $6.7 \mathrm{~mW}$ for $5 \mathrm{~min}$. The circle in (a) indicates the local annealed region. Reprinted from ref. 42 with permission.

(a)

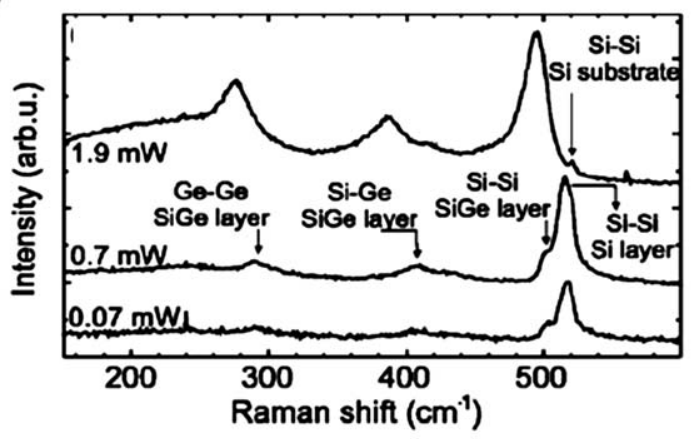

(b)

\section{Excitation power ( $\mathrm{mW})$}

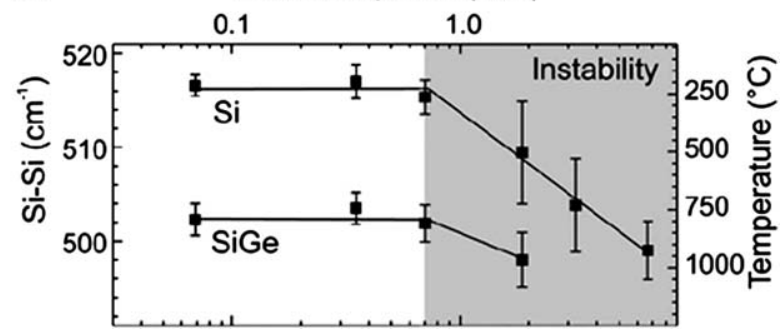

Fig. 21 Raman spectra of a single freestanding $9 \mathrm{~nm} \mathrm{Si}_{0.67} \mathrm{Ge}_{0.33} / 17 \mathrm{~nm}$ $\mathrm{Si}$ tube. (a) Raman spectra at excitation power ranging from 0.07 to $1.9 \mathrm{~mW}$. (b) Raman shifts of $\mathrm{Si}-\mathrm{Si}$ vibration mode in the $\mathrm{Si}$ and $\mathrm{SiGe}$ layers, respectively, as a function of excitation power and corresponding tube wall temperature. Reprinted from ref. 42 with permission.

mechanical properties of a nanocoil and a nanobelt has been performed recently via the micromanipulation technique. ${ }^{12,13,90}$ We briefly describe the mechanical characterization here.

(a)

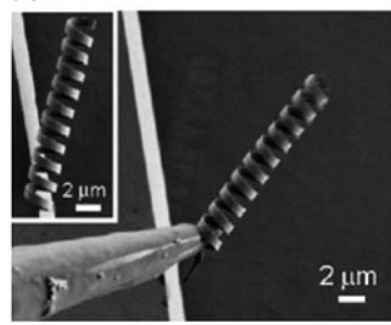

(c)
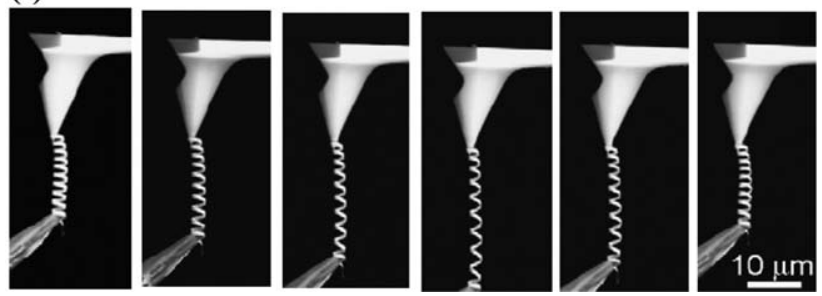

Fig. 22 SEM images for picking up and manipulation processes of a nanocoil created from the $11 \mathrm{~nm} \mathrm{SiGe/8} \mathrm{nm} \mathrm{Si/21} \mathrm{nm} \mathrm{Cr} \mathrm{trilayer.} \mathrm{(a} \mathrm{and}$ b) An as-fabricated nanocoil (inset of a) is picked up by a $\mathrm{W}$ probe and soldered to the tip of an AFM cantilever. Inset to (b) is an enlarged image. (c) Stiffness characterization via stretching and relaxing the nanocoil between the $\mathrm{W}$ tip and AFM cantilever (from left to right). All images have the same $10 \mu \mathrm{m}$ scale bar. Reprinted from ref. 12 with permission. 
The strained membranes roll into $3 \mathrm{D}$ nanostructures after release from the substrate. The rolled-up structures rest on or are still attached to the substrate. They can be cut off and picked up by a manipulator, for example, a sharp tungsten (W) probe, and soldered to an AFM cantilever using e-beam induced deposition in the SEM chamber, as shown in Fig. 22(a) and (b). A micromanipulator installed in a SEM chamber, equipped with an atomic force microscope (AFM) cantilever, allows in situ monitoring of the manipulation process.

The detailed soldering process can be found in ref. 12. By moving the $\mathrm{W}$ tip downward (upward), the nanocoil is elastically stretched (contracted). The applied force is measured by the AFM cantilever and the deformation of the nanocoil is recorded by SEM. The mechanical properties of a nanocoil rolled from a $11 \mathrm{~nm} \mathrm{SiGe} / 8 \mathrm{~nm} \mathrm{Si} / 21 \mathrm{~nm} \mathrm{Cr}$ multilayer have been investigated with such an experimental setup. ${ }^{12}$ Fig. 22(c) shows a series of SEM images of the manipulation process. The durability of the nanocoil is tested by repeating the pulling process for several cycles. No damage to the nanocoil is observed. The elongation length with respect to applied force reveals that a linear relationship between applied force and elongation exists until the spring is extended to $91 \%$ of its original length, indicating the excellent elasticity of the nanocoil. A spring constant of $0.003 \mathrm{~N} / \mathrm{m}$ for this nanocoil is obtained by linear fitting of experimental data. ${ }^{\mathbf{1 2}}$ This spring constant is an order of magnitude smaller than that of the most flexible available AFM cantilever $\left(\sim 10^{-2} \mathrm{~N} / \mathrm{m}\right)$. Such nanocoils are expected to be useful as ultrasensitive force sensors.

The radial stiffness of a multiwalled $\mathrm{Si} / \mathrm{Cr}$ nanoring has also been investigated using the same method. ${ }^{13}$ The enlarged SEM image in Fig. 23 shows no plastic deformation and sliding between interlayers, indicating the multi-walled nanoring remains closed during the pulling process. The elongation with respect to external load is plotted in Fig. 23. The spring constant of a 2.5 -turn $\mathrm{Si} / \mathrm{Cr}(35 / 10 \mathrm{~nm})$ nanoring is determined as $0.332 \mathrm{~N} / \mathrm{m}$ by linear fitting of experimental data. The stiffness of the nanoring is tunable by varying the number of rotations (wall thickness) and the ring width.

Another interesting study tests the interlayer bonding strength of a multi-walled nanoring by unrolling it using a manipulator

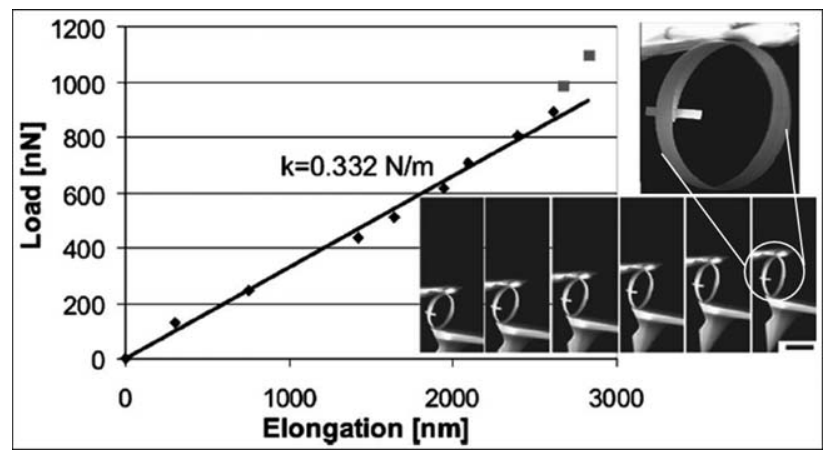

Fig. 23 Radial stiffness characterizations of single $35 \mathrm{~nm} \mathrm{Si} / 10 \mathrm{~nm} \mathrm{Cr}$ nanobelts. Inset shows a series of SEM images for pulling the nanoring from left to right with increasing external load. Zoomed-in image of the nanoring denoted in the circle shows no fracture occurs during the pulling. Reprinted from ref. 13 with permission. Image courtesy of Li Zhang. probe. ${ }^{90}$ SEM measurements reveal that the adjacent interlayers of a Si/Cr multi-walled nanoring are bonded, forming a closed ring. The strong interlayer bonding can be attributed to hydrogen bonds formed between bilayers, originating from the wet selective etching generally used in the releasing process. ${ }^{90}$

\subsection{Electrical properties}

The first electrical characterization of a rolled-up structure was performed in $2001 .^{61}$ The results showed a resistance of $10 \mathrm{k} \Omega$ for a highly boron doped $124 \mathrm{~nm}$-thick rolled-up SiGe/Si microspiral with a diameter of $13 \mu \mathrm{m} .{ }^{61} \mathrm{In}$ this work, the microspiral was detached by a manipulator from the $\mathrm{Si}$ substrate after release and transferred onto a new substrate with two metal contacts. Very recently, a novel device has been designed to integrate a freestanding microtube with two metal contacts, which facilitates a comprehensive electrical characterization of a freestanding microtube. ${ }^{17}$ Fig. 24 shows a device layout and SEM images of a freestanding tube connected to two contacts. The strained $\mathrm{Si}_{0.64} \mathrm{Ge}_{0.36} / \mathrm{Si}$ bilayer was highly boron doped $\left(\mathrm{Si}_{0.64} \mathrm{Ge}_{0.36}: \mathrm{B} / \mathrm{Si}: \mathrm{B}\right)$. It rolled into a tube after etching off the sacrificial layer (lightly doped $\mathrm{Si}$ ) via Approach 1 described in Sec. 3.3. The SEM images in Fig. 24 show a typical $10 \mathrm{~nm}$ $\mathrm{Si}_{0.64} \mathrm{Ge}_{0.36}: \mathrm{B} / 8 \mathrm{~nm} \mathrm{Si}: \mathrm{B}$ tube bridged over the substrate surface between the two designated contacts. A $10 \mathrm{~nm}$ Ti/200nm Au film was evaporated in the contact areas for the $\mathrm{Si}: \mathrm{B} / \mathrm{SiGe}: \mathrm{B}$ bilayer, forming ohmic contacts, which is crucial for a two-probe measurement.

The electrical characterization is performed by applying a voltage and measuring the current between the contacts. The current-voltage $(I-V)$ characteristic was measured for three different cases as shown in Fig. 25: before (dash-double dotted line) and after (solid line) rolling up a $10 \mathrm{~nm} \mathrm{Si}$ S. $_{04} \mathrm{Ge}_{0.36} / 8 \mathrm{~nm} \mathrm{Si}$ bilayer, and after the complete lift-off of the bilayer from the substrate (dashed line). Fig. 25 shows that the $I-V$ curve is linear in the whole range, both for the unreleased film and the rolled-up

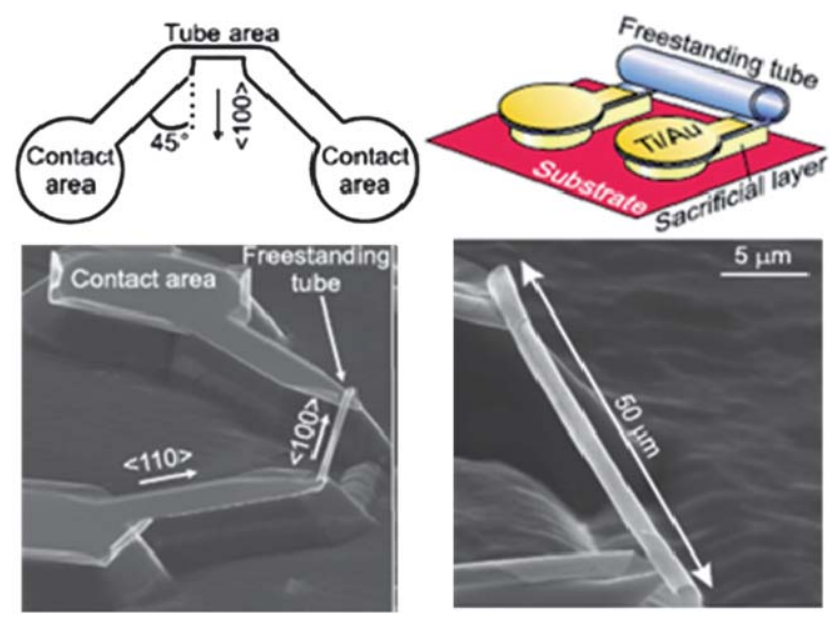

Fig. 24 A device with a freestanding $\mathrm{SiGe} / \mathrm{Si}$ tube connected to two contacts. Upper panel: A mask design for a tube bridged between two contacts and a schematic diagram of the device after etching the sacrificial layer; bottom panel: SEM images of a freestanding tube rolled from a $\mathrm{Si}_{0.64} \mathrm{Ge}_{0.36}$ : $\mathrm{B} / \mathrm{Si}$ : B bilayer bridging two contacts. Reprinted from ref. 17 with permission. 


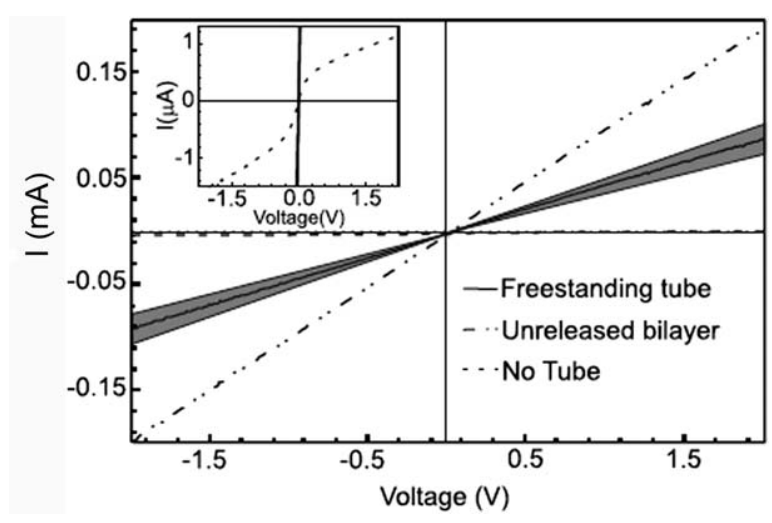

Fig. $25 I-V$ curves measured for a $\mathrm{Si}_{0.64} \mathrm{Ge}_{0.36}: \mathrm{B} / \mathrm{Si}: \mathrm{B}$ bilayer between the two contacts before the etching of the sacrificial layer: $R_{\text {asg }}=10 \mathrm{k} \Omega$ (dash-double dotted line) and after it, for a freestanding tube: $R_{\text {asg }}=$ $22 \mathrm{k} \Omega$ (solid line) and in the case that the bilayer is completely lifted off (dashed line). The inset is a magnification of the $I-V$ curve recorded after the removal of the tube from the substrate. Reprinted from ref. 17 with permission.

tube, a characteristic of ohmic contacts. The grey area represents the error bar $( \pm 3 \%)$ obtained for the $I-V$ curve of the rolled-up film by measuring fifteen tubes. The inset shows that the maximum current passing from one contact to the other through the substrate is about two orders of magnitude lower than the one measured prior to the complete release of the tube. The resistances $\left(R_{\text {asg }}\right)$ of the unreleased film and a rolled-up tube are 10 and $22 \mathrm{k} \Omega$, respectively, and the corresponding sheet resistances are 0.2 and $0.44 \mathrm{k} \Omega /$ square, respectively. These values are in fairly good agreement with the calculated values. The calculated sheet resistance for the unreleased bilayer with doping level $N=1.7 \times 10^{20} \mathrm{~cm}^{-3}$ is $0.33 \mathrm{k} \Omega /$ square, which corresponds to a resistance of $14 \mathrm{k} \Omega$ for the designed pattern. An increase of sheet resistance of the film after release from the substrate is observed. This may be explained by the influence of charging in surface states on the conductance. A high density of surface states that act as charge traps may be generated on the inner and outer surfaces of the tube wall, which are covered with a thin chemical oxide. A surface depletion layer grows with the surface charges, leading to a decrease of the effective conducting region of the tube wall. ${ }^{17}$

The tube resistance with respect to the thickness of the $\mathrm{SiGe} / \mathrm{Si}$ bilayer was also investigated. The dependence of the tube resistance on the bilayer thickness shows a monotonic decrease, from $110 \mathrm{k} \Omega$ for the thinnest film $(15 \mathrm{~nm})$ to $9 \mathrm{k} \Omega$ for a 30 -nm-thick layer. The tube resistance can be decrease further after rapid thermal annealing at a moderate temperature $\left(\sim 700{ }^{\circ} \mathrm{C}\right)$. The details of the measurement with respect to the tube thickness and annealing are described in ref. 17.

Electron transport in low-dimensional electron systems with respect to the magnetic field (magnetotransport) has been characterized in rolled-up III-V membranes. ${ }^{15,71}$ The results show that magnetotransport in freestanding curved/tubular membranes after release is quite different from that in its planar counterpart, still attached to the underlying substrate. The magnetoresistance in the nonplanar membranes is higher than in the planar ones at zero magnetic field and then increases further with applied magnetic field, but in a manner similar to the planar one. ${ }^{71}$ The initial rise may be attributed to an increase of electron scattering by surfaces. There exist dangling bonds at the bottom (inner) surface of tubular membranes after releasing from the host substrate by etching away the sacrificial layer. They may act as surface scattering centers and affect the electrical properties. The top surface is assumed to be the same as that of flat ones.

The magnetotransport in nonplanar $\mathrm{SiGe} / \mathrm{Si}$ membranes has been investigated as well. ${ }^{91}$ In this work, the low-temperature ballistic magnetoconductance is calculated in a curved $\mathrm{SiGe} / \mathrm{Si}$ resonant quantum cavity (RQC) under a low bias. Fig. 1 in ref. 91 shows a sketch of a curved $300 \times 300 \mathrm{~nm}^{2}$ RQC created on a $\mathrm{SiGe} / \mathrm{Si}$ bilayer membrane with a flat bottom tethered to the substrate. Narrow $(\sim 50 \mathrm{~nm})$ quantum point contacts connect the cavity to the source and drain leads. The $2 \mathrm{D}$ electron system is formed at the interface between the strained undoped Si and the relaxed n-type doped $\mathrm{SiGe}$. The detailed calculation can be found in ref. 91. The influence of curvature on electric transport with/ without the magnetic field is calculated.

Fig. 4 in ref. 91 shows that the conductance $\left(G_{0}=2 \mathrm{e} / \mathrm{h}\right)$ is very sensitive to the curvature $(\alpha)$ variation at a given sheet carrier density $\left(\mathrm{N}=4 \times 10^{10} \mathrm{~cm}^{-2}\right)$. In zero/low magnetic field, at lower curvatures, the carrier density is high at the drain-end quantum contacts, meaning there is a current flow between the source and the drain. As curvature increases, the coupling between the source and the drain decreases, reducing the conductance. The conductance can be modulated by changing the curvature while in the high magnetic field as well. ${ }^{91}$

\subsection{Optical properties}

Rolled-up tubes as building blocks for optical devices were proposed for the first time in $2006 .{ }^{15}$ The functionality of III-V based tubes was demonstrated via ring resonators and optical waveguides. In these early works, optical modes circulating in the tube cavity (i.e., whispering-gallery modes), and propagating along the tube axis were probed by the photoluminescence from low-density InAs quantum dots embedded in the tube wall. The same concept (i.e., incorporating an optically active material as an internal light source in the circular cavity) was applied to demonstrate the optical functionality of group IV based tubes. ${ }^{16,92}$ In both these studies, annealing of $\mathrm{Si} / \mathrm{SiO}_{\mathrm{x}}$ tubes is used to fabricate $\mathrm{Si}$ nanostructures integrated into a rolled-up solidstate matrix. The detailed description of the fabrication process can be found in ref. 16 .

The $\mathrm{Si} / \mathrm{SiO}_{\mathrm{x}}$ tubes undergo rapid thermal annealing at $850{ }^{\circ} \mathrm{C}$ for $30 \mathrm{~min}$ to activate a $\mathrm{Si} / \mathrm{SiO}_{2}$ phase separation in the Si-rich oxide, leading to the formation of $\mathrm{Si}$ agglomerates in the tube wall. The optical properties of a hybrid $\mathrm{Si} / \mathrm{SiO}_{\mathrm{x}}$ microtube have been investigated using fluorescence and microphotoluminesence ( $\mu$-PL) spectroscopy. SEM and XTEM images, shown in Fig. 26, demonstrate that $7 \mathrm{~nm} \mathrm{SiO}_{\mathrm{x}} / 8 \mathrm{~nm} \mathrm{Si}$ bilayers can roll into microtubes with multiple rotations, resulting in the formation of a radial $\mathrm{SiO}_{\mathrm{x}} / \mathrm{Si}$ superlattice in the tube wall. The XTEM image [Fig. 26(b)-top panel] shows the tube wall structure after the thermal-annealing step. The $\mathrm{SiO}_{\mathrm{x}} / \mathrm{Si}$ superlattice remains intact and a sharp $\mathrm{SiO}_{\mathrm{x}} / \mathrm{Si}$ interface is observed. Energy filtered TEM (EFTEM) is performed to reveal the oxygen content in the amorphous regions [see Fig. 26(b) bottom panel]. 


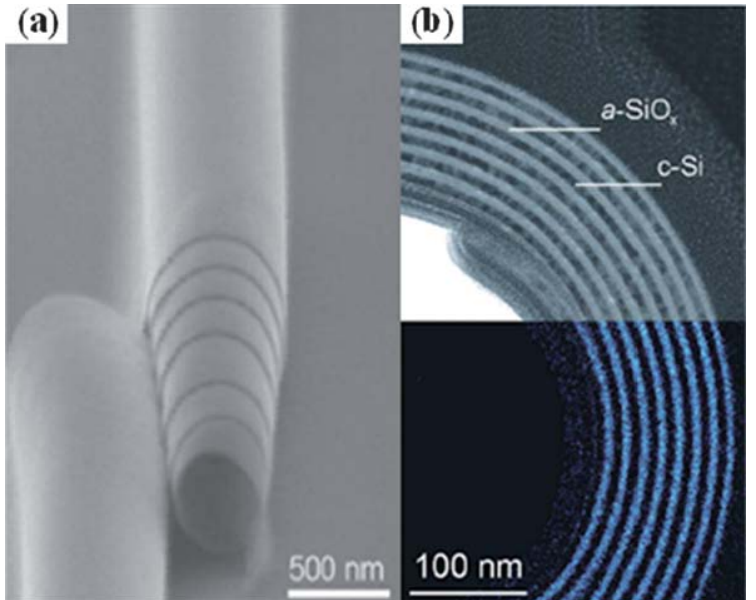

Fig. 26 A typical rolled-up tube from $7 \mathrm{~nm} \mathrm{SiO} / 8 \mathrm{~nm} \mathrm{Si}$. (a) $\mathrm{SEM}$ image. (b) XTEM image of a tube wall consisting of $7 \mathrm{~nm} a-\mathrm{SiO}_{\mathrm{x}}$ (bright area) and $8 \mathrm{~nm} c$-Si (dark area). False-color EFTEM of the $\mathrm{Si} / \mathrm{SiO}_{\mathrm{x}}$ tubewall illustrates the oxide content in the SiOx layer. Reprinted from ref. 16 with permission. Image courtesy of Oliver Schmidt.

$\mu$-PL spectroscopy is performed on $\mathrm{SiO}_{\mathrm{x}} / \mathrm{Si}$ rolled-up tubes at both $8 \mathrm{~K}$ and room temperature. A laser operating at $532 \mathrm{~nm}$ with a spot size of $\sim 2 \mu \mathrm{m}$ was used as a light source. A modulation of the PL spectrum appears in freestanding $\mathrm{SiO}_{x} / \mathrm{Si}$ tubes because of optical resonant modes occurring inside the tube wall as a certain wavelength of light circulates in the tube. The PL spectra at $8 \mathrm{~K}$ in Fig. 27 show that pronounced modes with different spacing depend on the tube diameter. The mode spacing is inversely proportional to the diameter of the resonator. Following the discussion in ref. 16, finite domain time difference (FDTD) simulations were performed to obtain both the optical resonant energies and the intensity pattern from the rolled-up tube structures. Modes are labeled according to their azimuthal $(M)$ and radial $(N)$ numbers. The refractive index of the $\mathrm{SiO}_{x}$ area is assumed as a constant value equal to 1.7. The refractive index of the $\mathrm{Si}$ area is varied as a function of wavelength. The simulated results are in good agreement with the experiment. Fig. 27 presents the intensity pattern of a mode at an energy of $1.95 \mathrm{eV}(M=20$ and $N=1)$. This pattern reveals that the light can be confined inside the tube wall despite its small thickness (40 $\mathrm{nm}$ ). Nevertheless, the limited tube wall thickness is probably the main reason for the linewidth broadening of the resonant modes. Based on FDTD simulations, it is expected that by increasing the number of rotations, the light losses can be reduced, leading to an improvement of the quality factor of the structure and a narrowing of the mode peaks.

Waveguiding along the axis of annealed $\mathrm{Si} / \mathrm{SiO}_{\mathrm{x}}$ tubes has also recently been demonstrated. ${ }^{92}$ Microtubes rolled from a $20 \mathrm{~nm}$ $\mathrm{Si} / 5 \mathrm{~nm} \mathrm{SiO}$ bilayer are investigated. The $\mathrm{Si} / \mathrm{SiO}_{\mathrm{x}}$ tubes undergo the previously described annealing process to promote formation of emitting Si nanoclusters in the tube wall. Tubes lying on the substrate or transferred onto a quartz plate are excited using a laser with wavelength of $532 \mathrm{~nm}$ focused to a $4 \mu \mathrm{m}$ spot by a $20 \mathrm{x}$ microscope objective. The emitted light is collected through the same objective and detected with a spectrometer equipped with a Si charge-coupled-device (CCD) camera. To study light emission and propagation along its whole length, the rolled-up tube is

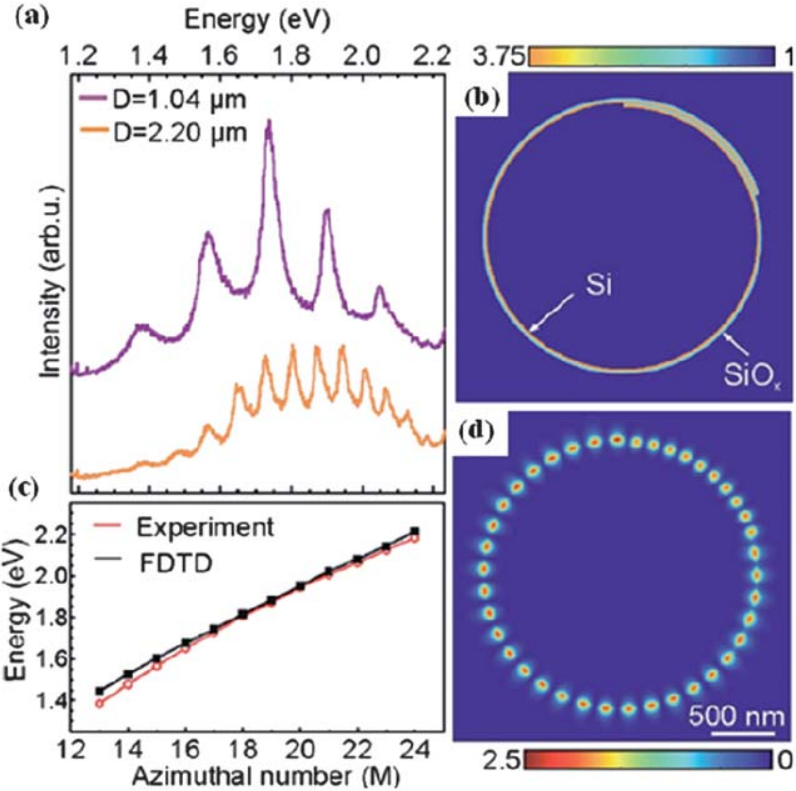

Fig. $27 \mathrm{PL}$ spectra at $8 \mathrm{~K}$ of a single freestanding $\mathrm{SiO} x / \mathrm{Si}$ microtube with diameters of $1.04 \mu \mathrm{m}$ (upper spectrum) and $2.2 \mu \mathrm{m}$ (lower spectrum). (b) Refractive-index contrast of the rolled-up tube used for the FDTD simulations. The tube wall consists of $20 \mathrm{~nm} \mathrm{SiO} x / 20 \mathrm{~nm}$ Si.(c) Energy positions of the modes extracted from the lower spectrum in (a) compared to the mode energies as a function of the azimuthal number $(M)$ obtained from FDTD simulation. (d) Intensity pattern of the resonant mode at $1.95 \mathrm{eV}$ with $M=20$ and $N=1$ from the structure shown in (b). Reprinted from ref. 16 with permission.

aligned with the entrance slit of the spectrometer. In this way the tube is imaged on the CCD camera, enabling spatially resolved spectroscopy. Motorized linear stages allow the tube to be translated with respect to the laser spot in the direction parallel and perpendicular to its axis.

Fig. 28 (a)-(c) displays PL spectra collected at the photoexcited region and at the tube exits when the laser beam is focused near the bottom end of a $80 \mu \mathrm{m}$-long tube with a $\sim 2 \mu \mathrm{m}$ diameter [see inset in Fig. 28(b)]. The modulation of the broad PL spectrum observed in Fig. 28(b) is caused by the resonant optical modes confined in the tube wall. Because modes are caused by constructive interference of light confined in a section of the tube, no modulation is observed for light propagating along the tube axis and escaping from the tube ends [Fig. 28(a) and (c)]. The spectra collected from those regions are red-shifted with respect to the spectrum collected from the region where the laser spot is focused. The measured red shift is attributed to light re-absorption in delocalized states of the nanostructured $\mathrm{Si}$, more pronounced at shorter-wavelength mode peaks. The asymmetry of the wide band emitted at the photoexcited point drastically changes for the PL signal acquired at the tube exits, as shown in Fig. 28.

Conventionally, optical emission from semiconductors is via the conduction-to-valence band optical transitions. Very recently, a novel radiation emission mechanism from wrinkled $\mathrm{Si}_{1-\mathrm{x}} \mathrm{Ge}_{\mathrm{x}} / \mathrm{Si}_{1-\mathrm{y}} \mathrm{Ge}_{\mathrm{y}}$ membranes has begun to be explored. ${ }^{93}$ The emission mechanism is based on the change of acceleration of carriers, when they travel along the sinusoidal trajectory in wrinkled $\mathrm{Si}_{1-\mathrm{x}} \mathrm{Ge}_{\mathrm{x}} / \mathrm{Si}_{1-\mathrm{y}} \mathrm{Ge}_{\mathrm{y}}$ membranes, a manner similar to 


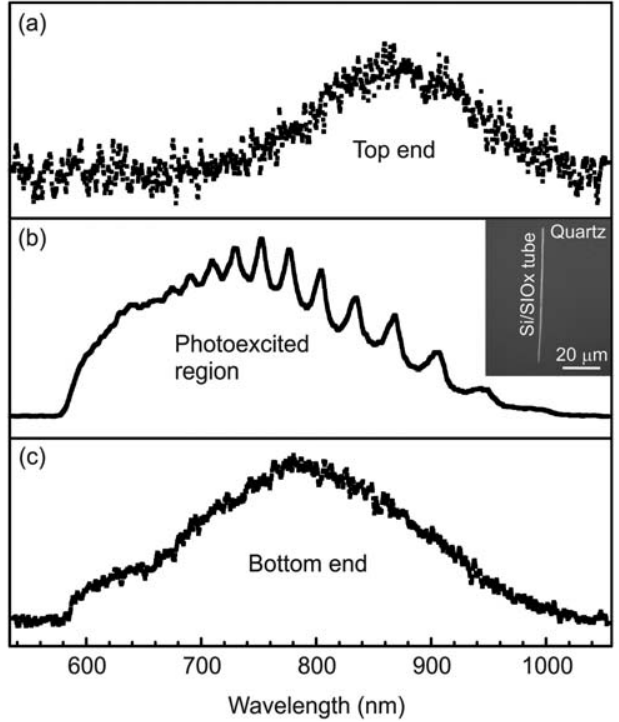

Fig. 28 PL spectra acquired from the top end (a), the photo excited region (b), and the bottom end (c) of a $80 \mu \mathrm{m}$-long tube with laser beam focused near the bottom end. The inset in (b) is an optical image of a typical tube on quartz. Reprinted from ref. 92 with permission.

synchrotron radiation with undulators, or like a free-electron laser. As shown in Fig. 29, as a voltage is applied across the periodically wrinkled structure, the carriers (holes in p-type $\mathrm{Si}_{1-\mathrm{x}} \mathrm{Ge}_{\mathrm{x}} / \mathrm{Si}_{1-\mathrm{y}} \mathrm{Ge}_{\mathrm{y}}$ wrinkled structures) change direction periodically. The acceleration of carriers will change correspondingly, resulting in emission of radiation. The same effect is expected to occur in n-type wrinkled membranes.

The theoretical analysis demonstrates that the emission wavelength covers a wide spectrum, depending upon the periodicity of wrinkles $\left(L_{w}\right)$ and the velocity of holes in response to the electric field $(E)$. The velocity of carriers is proportional to the magnitude of electric field, i.e., $v=\mu E$, where $\mu$ is the mobility. Fig. 30 shows the emission wavelength $\left(\lambda^{\prime}\right)$ as function of $E$ for different $L_{w}$. $\lambda^{\prime}$ decreases as $E$ increases and/or $L_{w}$ decreases. The mobility is set to $1400 \mathrm{~cm}^{2} \mathrm{~V}^{-1} \mathrm{~s}^{-1}$. The detailed calculation is described in ref. 93. The calculated radiation power in the infrared (IR) range is in the order of milliwatts for a p-type $\mathrm{Si}_{0.51} \mathrm{Ge}_{0.49} / \mathrm{Si}_{0.82} \mathrm{Ge}_{0.18}$ wrinkled structure with $L_{w}$ of $0.1 \mu \mathrm{m}$. An optimal $L_{w}$ can be designed based on the design principles described in Sec. 2. Periodically wrinkled membranes may be the realization of a real-space undulator, and can serve as an electromechanically tunable broadband electromagnetic frequency source (the Blick-Lagally oscillator). ${ }^{94}$

\subsection{Surface properties}

Thinness, a unique feature of nanomembranes, creates a large surface-to-volume ratio. Nanomembranes are mechanically ultra-compliant as well, which make them sensitive to very small external forces. One can expect that modification of membrane surfaces may lead to the deformation of membranes. Very recently, local-wetting induced deformation of rolled-up $\mathrm{SiGe} / \mathrm{Si}$ membranes has been demonstrated. ${ }^{84}$ The SiGe/Si membranes are patterned and aligned to the $<100>$ crystalline orientation (the most compliant direction in $\mathrm{SiGe} / \mathrm{Si}$ ). The $\mathrm{Si}$ layer is

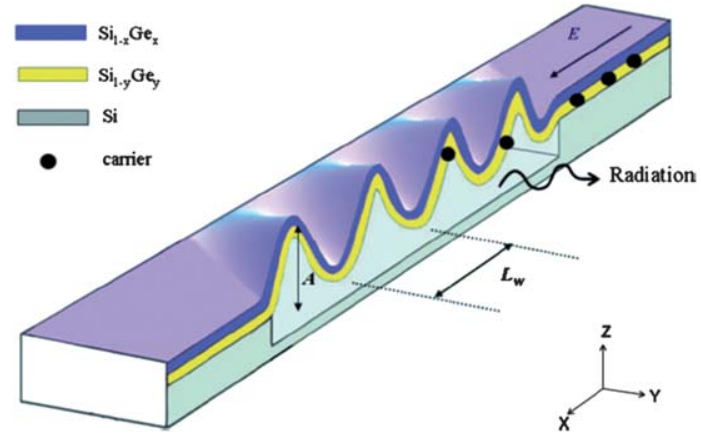

Fig. 29 Schematic illustration of a wrinkled structure. A voltage applied across the wrinkled structure leads to carriers (solid dots) moving in the wrinkled bilayer though a sinusoidal path. Reprinted from ref. 93 with permission.

epitaxially grown on SGOI to form the bilayer membrane and it is released from its host substrate via Approach 3 described in Sec 3. By careful control of the rate and uniformity of etching, a large array of freestanding $3 \mathrm{D}$ tubes can be achieved, as shown in Fig. 18.

As shown in Fig. 13 (a) and (b), two different tube shapes can be obtained by careful design of membrane patterns. The partially opened tubes are used to study the surface chemical modification of curved membranes. Acetone, dyed green, is chosen to modify the surface properties. A small amount of acetone is dropped on the partially opened tube. As acetone evaporates the surface tension on the tube surfaces changes correspondingly. The differing evaporation rates of acetone from top $(\mathrm{Si})$ and bottom ( $\mathrm{SiGe}$ ) surfaces of the tube causes an imbalance in surface tension, leading to a net force to open the tube. The opening process is recorded via video microscopy. Fig. 31(a)-(d) shows micrographs of time sequences of tube opening and recovery as acetone evaporates from its surfaces. The evaporation rate of acetone inside the tube can be represented as a wetting length $(L)$. Fig. 31(e) shows that the opening gap of the tube does not increase until five seconds and reaches a maximum value in six seconds, and then decrease to its initial

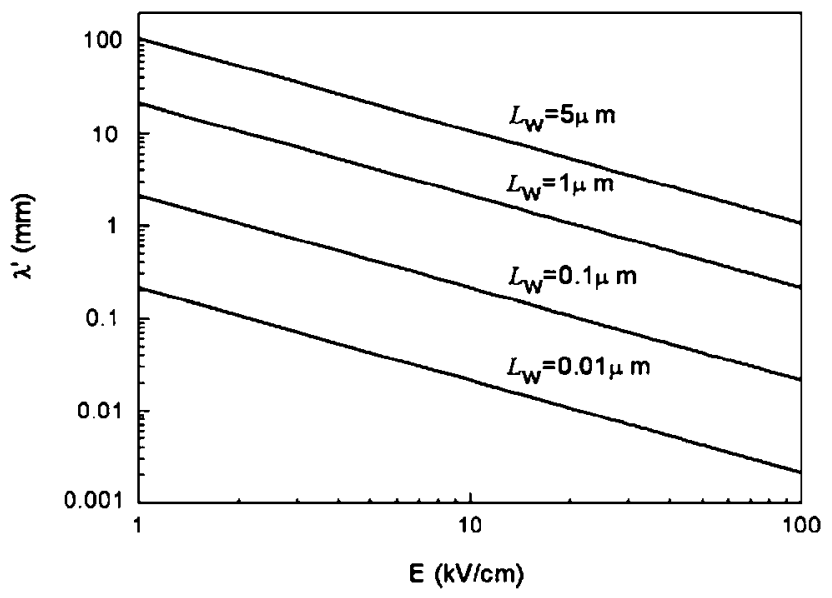

Fig. 30 Emission spectra with respect to the wrinkle periodicity and the electrical field. Reprinted from ref. 93 with permission. 
(a)

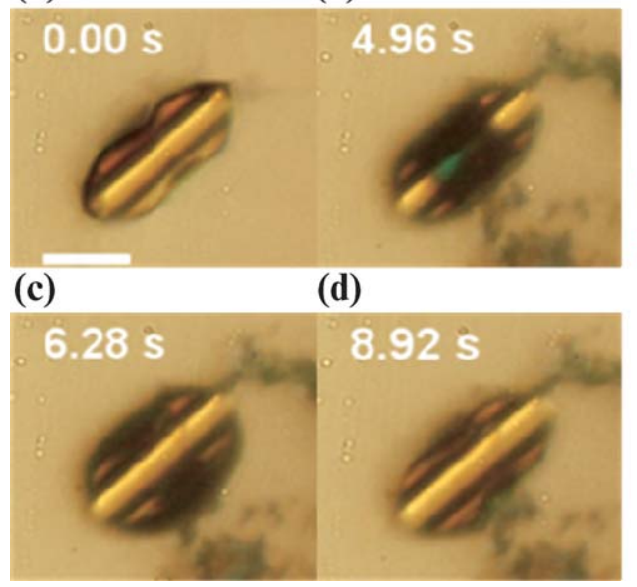

(e)

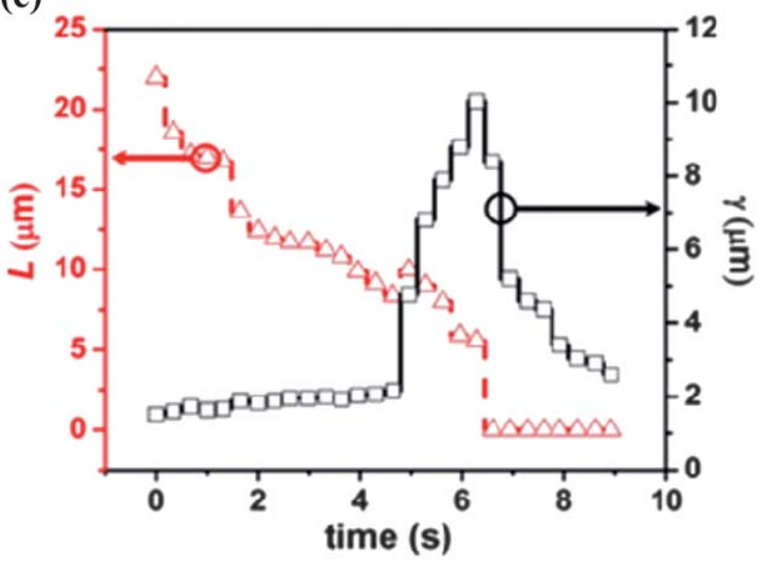

Fig. 31 Time-lapse sequences of a tube being opened and then recovering due to acetone evaporation on its surface. (a)-(d) Optical images. (e) Temporal changes of wetting length $L$ (left axis) and the opening gap $\gamma$ (right axis). Scale bar is $10 \mu \mathrm{m}$. Reprinted from ref. 84 with permission. Images courtesy of Minrui Yu.

gap, as $L$ decreases with time. The detailed description can be found in ref. 84.

\section{Potential applications of rolled-up and wrinkled membranes}

In Secs. 2 and 3, we described the design principles and fabrication techniques to roll up semiconductor and composite membranes into a variety of $3 \mathrm{D}$ nanostructures. In order to provide a backdrop for potential applications, we discussed materials properties in Sec. 4. In this section, we briefly enumerate some of the most promising applications.

\subsection{Microfluidics for biomedical applications}

A promising application of rolled-up microtubes is as microfluidic channels. ${ }^{15,18}$ The microtubes constrain the flow of liquids. Fluorescence measurements were performed to observe the liquid spread in the tubes. ${ }^{15,18}$ Because Si-based tubes are more readily integrated with modern $\mathrm{Si}$ technology, one can, in principle, position the microfluidic channel near electronic sensing circuits that can be used, for example, to count the flow of charged molecules through the tubes. Furthermore, the tubes may be envisioned as possible drug delivery systems in living organisms. Tubes can be made in quite small size by engineering the strain and film thickness of strained layers based on the design principles described in Sec. 2. Tubes with small diameter $(<20 \mathrm{~nm})$ can potentially be used for nanoinjection needles, nozzles for inkjet printing, and small-quantity fluid dispensers.

\subsection{Ultrasensitive force sensors}

Rolled-up nanosprings can have a very small spring constant, ${ }^{12}$ at least an order of magnitude smaller than that of the most flexible available AFM cantilever $\left(\sim 10^{-2} \mathrm{~N} / \mathrm{m}\right)$, as described in Sec. 4.2. Hence nanosprings are capable of sensing very small force with high accuracy and can be used as ultra-small force nanosensors/nanoactuators. The radial stiffness of a multiwalled
$\mathrm{Si} / \mathrm{Cr}$ nanoring has also been measured. For example, the spring constant of a 2.5-turn $\mathrm{Si} / \mathrm{Cr}(35 / 10 \mathrm{~nm})$ nanoring is determined as $0.332 \mathrm{~N} / \mathrm{m} .{ }^{13}$ The stiffness of a nanoring is tunable by varying the number of rotations (wall thickness) and the ring width, so nanorings may be usable as compact extended-range pressure sensors.

\subsection{Current-driven swinging micromirrors}

A micromirror device has been fabricated by release of a $\mathrm{SiGe} / \mathrm{Si}$ epitaxial bilayer from the underlying SOI substrate. ${ }^{95}$ The micromirror is supported by curved hinges. A gold conductive layer is deposited onto the bilayer for electrical characterization before the release step. The mechanical swinging of the micromirror is demonstrated by passing a current through it. Net angular displacements larger than 10 degrees are obtained at voltages smaller than $2 \mathrm{~V}$. The performance characteristics of these micromirrors are comparable to or better than the reported values for other MEMS optical switches or beam scanning devices.

\subsection{On-chip refractometric sensors}

Rolled-up $\mathrm{Si} / \mathrm{SiO}_{\mathrm{x}}$ miocrotubes can be used as refractometers. ${ }^{20}$ A $\mathrm{Si} / \mathrm{SiO}_{\mathrm{x}}$ microtube acts both as a natural pipe and as an optical sensor. Refractometry was tested by inserting a sugar solution into the microtube, leading to a change in refractive index and resulting in a shift of resonant-mode peak positions. $\mu$-PL spectroscopy using a laser was employed to characterize the microtube's optical response. Integrated PL intensity mapping was performed by scanning the laser over the microtube in submicron steps before and after the sugar solution was introduced into the microtube. These results reveal that spatial regions can be identified by air and solution modes, respectively. Comparing experimental data with simulations shows that the spectral sensitivity of this refractometer is remarkable. ${ }^{20}$ 


\subsection{Semiconductor optical emitter}

Emission of radiation from periodic wrinkled $\mathrm{Si}_{1-\mathrm{x}} \mathrm{Ge}_{\mathrm{x}} / \mathrm{Si}_{1-\mathrm{y}} \mathrm{Ge}_{\mathrm{y}}$ bilayer membranes has been proposed. ${ }^{93,94}$ When carriers move through a periodically wrinkled structure, the acceleration of carriers will change correspondingly, resulting in emission of radiation. The radiation frequency can cover a wide spectral range depending on the wavelength of wrinkles and velocity of carriers. A radiation power of $\mathrm{mWs}$ in the IR region is predicted for a microdevice containing a two-dimensional electron gas with a wrinkle wavelength of $0.1 \mu \mathrm{m}$.

\subsection{Remote chemical sensors}

A partially opened $\mathrm{Si} / \mathrm{SiGe}$ tube is mechanically very flexible, making it sensitive to tiny external-load changes on its surface. Such tubes can be used as chemical sensors if molecules selectively adsorb/desorb to/from the $\mathrm{Si} / \mathrm{SiGe}$ bilayer and cause an elastic deformation of the tube. ${ }^{84}$ Chemically functionalizing one side of the membrane can enhance selectivity.

\subsection{Advanced antenna architectures}

High-frequency lightweight antennas are needed for wireless communications ranging from consumer electronics to defense and space applications. Increasing the operating frequency of antennas from the $\mathrm{MHz}$ range into the $\mathrm{THz}$ range reduces signal loss and enhances efficiency. Conventionally, a metal coil fabricated by bending a metal strip is used for making antennas. A $\mathrm{THz}$ antenna requires the diameter of coil to be at the nanometer scale. Nanocoils created by rolling up nanomembrane strips are potential candidates for making $\mathrm{THz}$ antenna. Simulations reveal that nanocoil antennas can be operated at $\mathrm{THz}$ frequencies. ${ }^{96,97}$ The operating frequency can be tuned by varying the geometric parameters of nanocoils, such as the diameter, spacing, and number of turns. These parameters can be precisely controlled by the film thickness, length, and built-in strain. Nanocoil fabrication is compatible with standard semiconductor technology, making it is possible to fabricate antennas and other components of communication devices simultaneously and define their positions precisely.

\section{Conclusions}

In this review we retraced the successful story of strain-driven nanoarchitectures with a particular focus on group IV semiconductors. Templated self-assembly of nm-thick membranes into $3 \mathrm{D}$ objects combines top-down semiconductor processing and strain-driven actuation to fold 2D membranes into predictable shapes (e.g., tubes, helices, corner cubes, and periodic wrinkles) and configurations (e.g., wrinkled channel networks) on a substrate surface.

An additional value to the approach reviewed in the previous sections comes from the uniqueness of the result, i.e., nanostructures that combine the excellent properties of their constitutive materials (e.g., semiconductors or hybrid multilayer stacks) with their 3D geometry and ordered arrangement. Indeed, the work done in the past 10 years has demonstrated that the excellent structural, mechanical, electronic, optical, and surface properties of semiconductor thin films are not confined to planar configurations, but are preserved to a large extent in a $3 \mathrm{D}$ shaped membrane. The crystal structure of nanomembranes has proved to be unaffected by out-of-plane deformation for Si tubes with diameters of a few hundred nanometers or less, and good structural stability of various rolled-up objects (i.e., tubes, rings, and spirals) under mechanical and thermal stresses has been reported. These are significant results in the perspective of fabricating electronic, mechatronic, or photonic devices onto a 2D template before release from the substrate surface.

The hollow geometry of many of the nanomechanical architectures described in this review makes them ideal candidates for micro- and nanofluidics, optical ring resonators, and highfrequency radiation emitters. Both rolled-up tubes and periodic wrinkles are highly scalable in size, enabling a high tunability of resonator, emitter, or microfluidic-device response. The flexibility of nanomembranes also allows dynamic or post-processing changes of a 3D device response, even quite locally.

There is a message from the work of the last 10 years on nanoarchitectures. A century after Timoshenko analyzed the bending of bimetallic strips, fabrication of nanomechanical architectures has grown into a well established technology, yielding device structures with unique functionalities. Future research in this field may involve the integration of 3D devices implementing different functionalities on the same substrate and eventually on a transferable flexible support. Starting with 2D nanomembranes as a platform, the self-assembly into 3D nanoobjects in a controllable manner paves the way for exploring many potential additional applications of inorganic nanomembranes based on single-crystal semiconductors.

\section{Acknowledgements}

Work described in this review that was performed at the University of Wisconsin-Madison was supported by US DOE (Grant No. DE-FG02-03ER46028), by US NSF/MRSEC Program (DMR-0520527), and by US AFOSR (FA9550-09-10230). Work described in this review that was performed at the University of Utah was supported by US DOE (Grants No. DEFG02-03E46027 and DE-FG02-04ER46148). Preparation of this review was supported by AFOSR-MURI (Grant \#FA9550-08-10337). The authors would like to thank R. Blick and D. Savage for helpful discussions and thank M. Yu, Y.-W. Mo, E. Sutter, L. Zhang, O. Schmidt, and J. Rogers for the use of images.

\section{References}

1 Y.-W. Mo, D. E. Savage, B. S. Swartzentruber and M. G. Lagally, Phys. Rev. Lett., 1990, 65, 1020.

2 R. Nötzel, Semicond. Sci. Technol., 1996, 11, 1365.

3 K. Hiruma, M. Yazawa, K. Haraguchi, K. Ogawa, T. Katsuyama, M. Koguchi and H. Kakibayashi, J. Appl. Phys., 1993, 74, 3162.

4 C. Thelander, P. Agarwal, S. Brongersma, J. Eymery, L. F. Feiner, A. Forchel, M. Scheffler, W. Riess, B. J. Ohlsson, U. Gösele and

L. Samuelson, Mater. Today, 2006, 9, 28.

5 S. Iijima, Nature, 1993, 354, 56.

6 H. Engelkamp, S. Middelbeek and R. J. M. Nolte, Science, 1999, 284, 785.

7 V. Ya. Prinz, V. A. Seleznev, A. K. Gutakovsky, A. V. Chehovskiy, V. V. Preobrazhenskii, M. A. Putyato and T. A. Gavrilova, Phys. E., 2000, 6, 828 .

8 O. G. Schmidt and K. Eberl, Nature, 2001, 410, 168. 
9 H. Qin, N. Shaji, N. E. Merrill, H. S. Kim, R. C. Toonen, R. H. Blick, M. M. Roberts, D. E. Savage, M. G. Lagally and G. Celler, New J. Phys., 2005, 7, 241.

10 X. Li, J. Phys. D: Appl. Phys., 2008, 41, 193001.

11 M. Huang, C. Boone, M. Roberts, D. E. Savage, M. G. Lagally, N. Shaji, H. Qin, R. H. Blick, J. A. Nairn and F. Liu, Adv. Mater., $2005, \mathbf{1 7}, 2860$.

12 L. Dong, L. Zhang, D. J. Bell, D. Grützmacher and B. J. Nelson, J. Phys. Conf. Ser., 2007, 61, 257.

13 D. Grützmacher, L. Zhang, L. Dong, D. J. Bell, B. J. Nelson, A. Prinz and E. Ruh, Microelectron. J., 2008, 39, 478.

14 T. Kipp, H. Welsch, Ch. Strelow, Ch. Heyn and D. Heitmann, Phys. Rev. Lett., 2006, 96, 077403.

15 S. Mendach, R. Songmuang, A. Rastelli, M. Benyoucef and O. G. Schmidt, Appl. Phys. Lett., 2006, 88, 212113.

16 R. Songmuang, A. Rastelli, S. Mendach and O. G. Schmidt, Appl. Phys. Lett., 2007, 90, 091905.

17 F. Cavallo, R. Songmuang and O. G. Schmidt, Appl. Phys. Lett., 2008, 93, 143113; P. Zhang, E. Tevaarwerk, B.-N. Park, D. E. Savage, G. K. Celler, I. Knezevic, P. G. Evans, M. A. Eriksson and M. G. Lagally, Nature, 2006, 439, 703; S. A. Scott, W. Peng, A. M. Kiefer, H. Jiang, I. Knezevic, D. E. Savage, M. A. Eriksson and M. G. Lagally, ACS Nano, 2009, 3, 1683 .

18 A. A. Solovev, Y. F. Mei, E. Bermúdez Ureña, G. S. Huang and O. G. Schmidt, Small, 2009, 5, 1688.

19 G. S. Huang, Y. F. Mei, D. J. Thurmer, E. Coric and O. G. Schmidt, Lab Chip, 2009, 9, 263.

20 A. Bernardi, S. Kiravittaya, A. Rastelli, R. Songmuang, D. J. Thurmer, M. Benyoucef and O. G. Schmidt, Appl. Phys. Lett., 2008, 93, 094106.

21 Y. Forterre, J. M. Skotheim, J. Dumais and L. Mahadevan, Nature, $2005, \mathbf{4 3 3}, 421$

22 R. Abermann, Thin Solid Films, 1984, 115, 185.

23 J. A. Thornton, Thin Solid Films, 1989, 171, 5.

24 F. Elstner, C. Gautier, O. Piot, G. Contoux, F. Cosset, F. Nardou and J. Machet, Phys. Status Solidi A, 1996, 154, 669.

25 Y. F. Mei, G. S. Huang, A. A. Solovev, E. Bermúdez Ureña, I. Moench, F. Ding, T. Reindl, R. K. Y. Fu, P. K. Chu and O. G. Schmidt, Adv. Mater., 2008, 20, 4085.

26 V. Luchnikov, O. Sydorenko and M. Stamm, Adv. Mater., 2005, 17, 1177.

27 Ch. Deneke, R. Songmuang, N. Y. Jin-Phillipp and O. G. Schmidt, J. Phys. D: Appl. Phys., 2009, 42, 103001.

28 Y. Mei, D. J. Thurmer, F. Cavallo, S. Kiravittaya and O. G. Schmidt, Adv. Mater., 2007, 19, 2124.

29 P. Cendula, S. Kiravittaya, Y. F. Mei, C. Deneke and O. G. Schmidt, Phys. Rev. B: Condens. Matter Mater. Phys., 2009, 79, 085429.

30 F. Liu, M. G. Lagally and J. Zang, MRS Bull., 2009, 34, 190.

31 M. Huang, P. Rugheimer, M. G. Lagally and Feng Liu, Phys. Rev. B. Condens. Matter Mater. Phys., 2005, 72, 085450.

32 F. Liu, P. Rugheimer, E. Mateeva, D. E. Savage and M. G. Lagally, Nature, 2002, 416, 498.

33 F. Liu, M. Huang, P. Rugheimer, D. E. Savage and M. G. Lagally, Phys. Rev. Lett., 2002, 89, 136101.

34 S. Timoshenko, J. Opt. Soc. Am., 1925, 11, 233.

35 J. Zang and F. Liu, Appl. Phys. Lett., 2008, 92, 021905.

36 J. Zang, M. Huang and F. Liu, Phys. Rev. Lett., 2007, 98, 146102.

37 A. Cho, Science, 2006, 313, 164.

38 A. Malachias, C. Deneke, B. Krause, C. Mocuta, S. Kiravittaya, T. H. Metzger and O. G. Schmidt, Phys. Rev. B: Condens. Matter Mater. Phys., 2009, 79, 035301.

39 N. Y. Jin-Philipp, J. Thomas, T. Kelsch, R. Songmuang, Ch. Deneke and O. G. Schmidt, Appl. Phys. Lett., 2006, 88, 033113.

40 S. Mendach, S. Kiravittaya, A. Rastelli, M. Benyoucef, R. Songmuang and O. G. Schmidt, Phys. Rev. B: Condens. Matter Mater. Phys., 2008, 78, 035317.

41 D. J. Thurmer, C. Deneke, Y. Mei and O. G. Schmidt, Appl. Phys. Lett., 2006, 89, 223507.

42 R. Songmuang, N. Y. Jin-Phillipp, S. Mendach and O. G. Schmidt, Appl. Phys. Lett., 2006, 88, 021913.

43 Y. F. Mei, S. Kiravittaya, M. Benyoucef, D. J. Thurmer, T. Zander, C. Deneke, F. Cavallo, A. Rastelli and O. G. Schmidt, Nano Lett., 2007, 7, 1676.

44 G. G. Stoney, Proc. R. Soc. London, Ser. A, 1909, 82(553), 172-178.

45 J. J. Wortman and R. A. Evans, J. Appl. Phys., 1965, 36, 153.
46 P. G. Evans, D. S. Tinberg, M. M. Roberts, M. G. Lagally, Y. Xiao, B. Lai and Z. Cai, Appl. Phys. Lett., 2005, 87, 073112.

47 H.-J. Kim-Lee, D. E. Savage, C. S. Ritz, M. G. Lagally and K. T. Turner, Phys. Rev. Lett., 2009, 102, 226103.

48 L. Zhang, E. Ruh, D. Grützmacher, L. Ding, D. J. Bell, B. J. Nelson and C. Schönenberger, Nano Lett., 2006, 6, 1311.

49 A. I. Fedorchenko, A.-B. Wang, V. I. Mashanov, W.-P. Huang and H. H. Cheng, Appl. Phys. Lett., 2006, 89, 043119.

50 W.-P. Huang, H. H. Cheng, A. I. Fedorchenko and A.-B. Wang, Appl. Phys. Lett., 2007, 91, 053103.

51 R. Huang and Z. Suo, J. Appl. Phys., 2002, 91, 1135.

52 V. Ya. Prinz, V. A. Seleznev, A. V. Prinz and A. V. Kopylov, Sci. Technol. Adv. Mater., 2009, 10, 034502.

53 E. Cerda and L. Mahadevan, Phys. Rev. Lett., 2003, 90, 074302.

54 J. Zang and F. Liu, Nanotechnology, 2007, 18, 405501.

55 D. Yu and F. Liu, Nano Lett., 2007, 7, 3046.

56 S. V. Golod, V. Ya. Prinz, P. Wagli, L. Zhang, O. Kirfel, E. Deckhardt, F. Glaus, C. David and D. Grützmacher, Appl. Phys. Lett., 2004, 84, 3391.

57 Ch. Deneke, C. Muller, N. Y. Jin-Phllipp and O. G. Schmidt, Semicond. Sci. Technol., 2002, 17, 1278.

58 R. Songmuang, Ch. Deneke and O. G. Schmidt, Appl. Phys. Lett., 2006, 89, 223109.

59 Ch. Deneke and O. G. Schmidt, Phys. E., 2004, 23, 269.

60 N. Shaji, C. B. Simmons, M. Thalakulam, L. J. Klein, H. Qin, H. Luo, D. E. Savage, M. G. Lagally, A. J. Rimberg, R. Joynt, M. Friesen, R. H. Blick, S. N. Coppersmith and M. A. Eriksson, Nat. Phys., $2008,4,540$.

61 S. V. Golod, V. Ya Prinz, V. I. Mashanov and A. K. Gutakovshy, Semicond. Sci. Technol., 2001, 16, 181.

62 F. Wang, Y. Shi, J. Liu, Y. Lu, S. Gu and Y. Zhang, J. Electrochem. Soc., 1997, 144, L37.

63 O. G. Schmidt and N. Y. Jin-Phillipp, Appl. Phys. Lett., 2001, 78, 3310 .

64 L. Zhang, E. Deckhardt, A. Weber, C. Schönenberger and D. Grützmacher, Nanotechnology, 2005, 16, 655.

65 L. Zhang, E. Deckardt, A. Weber, C. Schönenberger and D. Grützmacher, Microelectron. Eng., 2006, 83, 1233.

66 A. Vorob'ev, P. O. Vaccaro, K. Kubota, T. Aida, T. Tokuda, T. Hyayshi, Y. Sakano, J. Ohta and M. Nunoshita, J. Phys. D: Appl. Phys., 2003, 36, L67.

67 F. Cavallo, R. Songmuang, C. Ulrich and O. G. Schmidt, Appl. Phys. Lett., 2007, 90, 193120.

68 F. Cavallo, W. Sigle and O. G. Schmidt, J. Appl. Phys., 2008, 103, 116103.

69 P. O. Vaccaro, K. Kubota and T. Aida, Appl. Phys. Lett., 2001, 78, 2852.

70 Ch. Deneke and O. G. Schmidt, Phys. E., 2004, 23, 269-273.

71 N. Shaji, H. Qin, L. J. Klein, C. Deneke, O. G. Schmidt and R. H. Blick, Appl. Phys. Lett., 2007, 90, 042101.

72 I. S. Chun and X. Li, IEEE Trans. Nanotechnol., 2008, 7(4), 493.

73 J. Q. Hu, Y. Bando, J. H. Zhan, M. Y. Liao, D. Golberg, X. L. Yuan and T. Sekiguchi, Appl. Phys. Lett., 2005, 87, 113107.

74 X. Chen, Z. Y. Zhang and M. G. Lagally, Phys. Rev. Lett., 1994, 73, 850.

75 F. Wu, X. Chen, Z. Y. Zhang and M. G. Lagally, Phys. Rev. Lett., 1995, 74, 574.

76 I. N. Stranski and V. L. Krastanov, Abhandlungen der MathematischNaturwissenschaftlichen Klasse. Sitzungsberichte der Akademie der Wissenschaften in Wien, Math.-naturwiss. Kl. IIb, 1939, 146, 797.

77 Y. Fujikawa, K. Akiyama, T. Nagao, T. Sakuri, M. G. Lagally, T. Hashimoto, Y. Morikawa and K. Terakura, Phys. Rev. Lett., 2002, 88, 176101

78 F. K. LeGoues, B. S. Meyerson, J. F. Morar and P. D. Kirchner, J. Appl. Phys., 1992, 71, 4230.

79 J. Tersoff and F. K. LeGoues, Phys. Rev. Lett., 1994, 72, 3570.

80 J. W. Matthews and A. E. Blakeslee, J. Cryst. Growth, 1974, 27, 118.

81 D. C. Houghton, J. Appl. Phys., 1991, 70, 2136.

82 C. Euaruksakul, M. M. Kelly, B. Yang, D. E. Savage, M. G. Lagally and G. K. Celler, ACS Nano, submitted.

83 Y. Zhang, M. Yu, D. E. Savage, M. G. Lagally, R. H. Blick and F. Liu, Appl. Phys. Lett., 2010, 96, 111904.

84 M. Yu, M. Huang, D. E. Savage, M. G. Lagally and R. H. Blick, IEEE Trans. Nanotechnol., 2010, DOI: 10.1109/ TNANO.2010.2066986. 
85 T. Tezuka, N. Sugiyama and S. Takagi, Appl. Phys. Lett., 2001, 79, 1798.

86 S. Nakaharai, T. Tezuka, N. Sugiyama, Y. Moriyama and S. Takagi, Appl. Phys. Lett., 2003, 83, 3516.

87 D. Khang, H. Jiang, Y. Huang and J. A. Rogers, Science, 2006, 311, 208

88 S. Tanaka, C. C. Umbach, J. M. Blakely, R. M. Tromp and M. Mankos, Appl. Phys. Lett., 1996, 69, 1235.

89 V. Zielasek, F. Liu, Y. Zhao, J. B. Maxson and M. G. Lagally, Phys. Rev. B: Condens. Matter Mater. Phys., 2001, 64, 201320.

90 L. Zhang, L. Dong and B. J. Nelson, Appl. Phys. Lett., 2008, 92, 143110.

91 G. J. Meyer, N. L. Dias, R. Blick and I. Knezevic, IEEE Trans. Nanotechnol., 2007, 6, 446.
92 F. Cavallo, R. Songmuang, A. Rastelli, M. Benyoucef and O. G. Schmidt, Proceedings of the IV Conference of Group IV Photonics, San Francisco, 9-11 Sept 2009.

93 A. I. Fedorchenko, H. H. Cheng, G. Sun and R. A. Soref, Appl. Phys. Lett., 2010, 96, 113104.

94 R. Blick and M. G. Lagally, patent applied for, 2007.

95 T. Tokuda, Y. Sakano, D. Mori, J. Ohta, M. Nunoshita, P. O. Vaccaro, A. Vorob'ev, Kubota and N. Saito, Electron. Lett., 2004, 40, 1333.

96 Y. M. Senousy, E. V. Moiseva and C. K. Harnett, University Government Industry Micro/Nano Symposium (UGIM), Louisville, KY, July 2008 13-16.

$97 \mathrm{M}$. Yu and R. H. Blick, private communication. 